\title{
HACIA UNA TIPOLOGÍA DE LA VARIACIÓN GRAMATICAL EN SOCIOLINGÜÍSTICA DEL ESPAÑOL*
}

Larga ha sido y es la polémica acerca de la posibilidad de extender el estudio de la variación al terreno de la sintaxis. Muchos son los problemas metodológicos y teóricos de ese propósito. La dificultad principal es la forma de constituir las variables que se vayan a estudiar. Si no hay equivalencia semántica entre las supuestas variantes sintácticas, mal se puede hablar entonces ni de variables ni de variantes, se ha dicho'. Se ha sugerido también que el primer paso para poder extender la variable sociolingüística a la sintaxis es estudiar detalladamente cuál es la función de una determinada variante en el discurso ${ }^{2}$. En el sentido más

* Agradezco las valiosas sugerencias de Sergio Bogard, que leyó un borrador de este trabajo. Todos los errores son, obviamente, míos.

${ }^{1}$ LABOV señaló que las "social and stylistic variation presuppose the option of saying "the same thing" in several different ways: that is, the variants are identical in referential or truth value, but opposed in their social and/or stylistic significance" (1972, p. 271). Conocida es la polémica suscitada a partir del trabajo leído por Lavandera en 1977 y publicado en 1978, también recogido en versión española en LAVANDERA 1984, pp. 37-46. Lavandera resume así los argumentos de su crítica: en las variables no fonológicas no es clara la noción de "decir lo mismo", no basta con los criterios de verdad; por ello mismo se hace problemática la noción de lo que "podría haber ocurrido"; si no cuenta la influencia extralingüística en un fenómeno, estamos, en realidad, ante una nueva noción de variable; el temor a poner en duda la "igualdad" ha hecho que se rechace que grupos o situaciones puedan tener necesidades diferentes que condicionen la selección de los significados transmitidos (1984, pp. 15-16). La polémica continuó en los trabajos de LABOV 1978 y Romaine 1981. Originó diversas tomas de posición (véase Lavandera 1984 , p. 15 , n. 2). Para completar la visión del problema, cf. Cheshire 1987; Milroy 1987, pp. 143-170; Sankoff 1988, esp. pp. 152155; JACOBSON 1989; SERRANO 1993; una buena reseña puede encontrarse en López MORALES 1989, pp. 91-105.

${ }^{2}$ Existen "dificultades inherentes a la extensión del concepto de «va- 
ambicioso, se ha defendido la importancia cognitiva del enfoque variacionista, que más bien podría sacar partido, precisamente, de las diferencias de significado ${ }^{3}$. En cualquier caso, en la naturaleza del tipo de variación a que se suele hacer referencia cuando se habla de "variación sintáctica", se sabe que pueden ser influyentes razones de índole fónica, morfológica, semántica, pragmática... y desde luego sintáctica. Parece entonces más aconsejable hablar de "variación gramatical" - entendiendo la gramática en sentido amplio-, como procuraremos hacer aquí. Como suelen ser muy diversos y mezclados los tipos de reglas que contribuyen a explicar aun el problema aparentemente más sencillo, se impone reflexionar sobre la "escala" a que vamos a observar los llamados fenómenos de variación gramatical't.

\section{LA ESCALA DE OBSERVACIÓN DE LA VARIACIÓN GRAMATTCAL}

Si se toma la decisión de estudiar alguna parte de la organización de la variación gramatical de una lengua, se topa de inmediato con el problema de la escala de observación, es decir, si tomamos

riable sociolingüistica" al nivel de la sintaxis. . Esta extensión requiere antes que nada un estudio riguroso de la función de cada variante en el discurso. Luego, el establecimiento de correlaciones sociolingüísticas válidas deberá hacerse con respecto a cada una de las funciones de las variantes" (SiLvACORVALÁN 1989, p. 128).

${ }^{3}$ Delbecoue 1990, 1991, 1992: "La aproximación que tiene en cuenta la variación omnipresente en el lenguaje podría denominarse "variacionista". No excluye el concepto de lengua, pero lo reconstituye en un marco cognitivo ya que considera la variación como una propiedad fundamental de los sistemas lingüísticos, que corresponden a las estructuras mentales que expresan y hacen comunicables hasta cierto punto... El estudio de la variación no se limita a la alternancia de formas que no entraña ninguna alteración semántica. Sólo de formas fonéticas o morfofonológicas competidoras puede pensarse que mantienen la equivalencia semántica una respecto de otras" (1992, p. 64).

4 Partimos del supuesto de que la variación no afecta de la misma manera a los diversos componentes de la lengua, en parte por la propia naturaleza de los fenómenos, en parte por el diferente control que un hablante puede ejercer sobre las varias clases de reglas sociolingüísticas (MARTín BUTRAGUEÑo, en prensa). Partimos de la idea de que un hablante quiere decir algo, que quiere decirlo de la forma más conveniente a sus propósitos, y que quiere decirlo para algo, aunque tenga limitaciones de diverso orden a su libertad lingüística. 
un texto cualquiera, es probable que, a simple vista, aparezca un cúmulo de fenómenos "variables". Observemos (1):

(1) Ése es el jugador de que yo te hablé, a quien conocí en el avión de regreso

Para empezar, podríamos fijarnos en la alternancia entre relativos que / quien; pero también podría elaborarse la lista de otro modo: ése $e_{\mathrm{i}}$, jugador $\mathrm{r}_{\mathrm{i}}, q u e_{\mathrm{i}}$, quien $_{\mathrm{i}}$, atendiendo a todos los elementos correferentes. Es más, podríamos fijarnos en (1) en muchos otros fenómenos: así, ¿por qué o para qué aparece una perífrasis de relativo del tipo ése es el jugador de que yo te hablé, y no otra fórmula, como de ese jugador es de quien yo te hablé? ¿Por qué o para qué aparece un sujeto yo en la primera oración, y no en la segunda? ¿Son todos estos hechos comparables? Si adoptamos una perspectiva muy generosa, podríamos preguntarnos incluso si un estudio de variación gramatical podría ocuparse de ejemplos como

$$
\text { Pepe }_{i} \text { fue a ver si querían contratarlo }
$$

Obviamente, hay "variación" entre $P e p e_{\mathrm{i}}$ y $l_{o_{\mathrm{i}}}$; si quisiéramos, con un ejercicio de imaginación metódica, ignorar el hecho de que existe una parcela de la gramática que ya estudia este fenómeno, podríamos llegar a preguntarnos si las reglas ordenadoras de este tipo de hechos son o no parte de una "teoría de la variación gramatical"'5. Porque, en realidad, no parece que esta teoría, tan vagamente formulada, se haya preocupado demasiado formalmente por saber con qué clase de reglas le concierne trabajar, más allá de un "variable" sentido común.

Es más, llevando las cosas al extremo, puede imaginarse una tal teoría de la variación que tratara, llanamente, de explicar por qué varían las formas de expresión de las infinitas oraciones pronunciables por cualquier hablante (lo que subsumiría la gramática en esta concepción dilatada del terreno de la variación). En el extremo opuesto, podría imaginarse una esquilmada sección descriptiva (a la que difícilmente podría llamarse "teoría" de nada)

${ }^{5}$ No estamos pensando ahora para nada en la teoría generativista de variación paramétrica; véase al respecto, por ejemplo, BRUCART 1987; RiemsdjK y Williams 1990, esp. pp. 247, 308, 398; ConTreras 1992, pp. 149-154. Un desarrollo muy interesante es el elaborado por D'INTRONo en 1982 (aunque publicado en 1987), con ejemplos de dialectos del español. En una tradición tipológica diferente, debe verse COMRIE 1989 (1981). 
que se ocupara de proporcionar documentaciones de fenómenos como los de (3):

(3) a. Pienso que vendrá

b. Pienso de que vendrá

Es más, esta sección podría limitarse a la descripción, digamos, de qué niveles de población emplean (3a) o (3b) con más frecuencia.

Claro está que otra solución a los problemas es decir que el estudio de la variación sintáctica es la "perspectiva" que intenta enfrentar hechos inicialmente confusos, como los que se desprenden de (4):

(4) a. Quiero ver a Juan

b. A Juan lo quiero ver

c. A Juan es a quien quiero ver

d. A Juan, quiero verlo,

con todos los medios posibles, para desgajar a lo largo de la investigación qué de sintáctico o qué de pragmático, etc., se reparten la explicación de estas construcciones. Sin embargo, planteadas así las cosas, podría no verse por ningún lado la necesidad de hablar de "variación" y, desde luego, mucho menos, de "teoría de la variación" 6 .

¿Es posible, entonces, una perspectiva o teoría variacionista (de la gramática) en sí misma? A estas alturas, parece necesario aclarar qué se entiende aquí por "variacionismo":

${ }^{6}$ Toda esta discusión podrfa parecer algo trivial, de no ser porque se ha planteado el peso cognitivo que pueden tener los fenómenos variables. Los planteamientos que pueden encontrarse en trabajos como los de Delbecque encuentran quizá fundamento en ideas como éstas: "Grammatical structure is based on conventional imagery, which reflects our ability to construe a conceived situation in alternate ways. The full conceptual or semantic value of a conceived situation is a function of not only its content (to the extent that one can speak of content apart from construal), but also how we structure this content with respect to such matters as attention, selection, figure/ground organization, viewpoint, and level of schematicity. In regard to all of these we are capable of making adjustments, thereby transforming one conceptualization into another that is roughly equivalent in terms of content but differs in how this content is construed. This is particularly evident in the case of autonomous processing, but is true for peripherally connected experience as well"' (LANGACKER 1987, p. 138). 
(5) "Variacionismo" es la teoría o perspectiva que defiende que el argumento cuantitativo tiene alguna importancia en el estudio del lenguaje ${ }^{7}$

Debe aclararse que (5) está mediatizado por la definición de “"argumento cuantitativo", tal como se hace en (6):

(6) Argumento cuantitativo es, al menos,

a. El que se apoya en la asignación de cantidades $x_{1}, x_{2} \ldots x_{n}$ a variantes $\left[y_{1}\right],\left[y_{2}\right] \ldots\left[y_{n}\right]$ de una variable $\langle y\rangle:\left[\nu_{\mathrm{i}}\right] \rightarrow x_{\mathrm{i}}$

b. El que se construye asignando jerarquías entre variantes $\left[y_{1}\right],\left[y_{2}\right] \ldots\left[y_{\mathrm{n}}\right]$ de una variable $\langle y\rangle$. Por ejemplo, $\left[y_{3}\right]>\left[y_{1}\right]>\left[y_{2}\right]$

c. El que se construye asignando valores de presencia [1] o ausencia $[\emptyset]$ a las variantes $\left[y_{\mathrm{I}}\right],\left[y_{2}\right] \ldots\left[y_{\mathrm{n}}\right]$ de una variable $\langle y\rangle$. Por ejemplo, $\left[y_{1}\right] \rightarrow[1] ;\left[y_{2}\left[\rightarrow[\emptyset] ;\left[y_{3}\right] \rightarrow\right.\right.$ [1]

Con esta definición, es fácil deducir que una regla interesante para el "variacionismo" será cualquier regla para la que pueda defenderse que el argumento cuantitativo tiene algún peso en su constitución.

El problema de la escala de observación, entonces, viene a ser el de qué clase de reglas son las adecuadas para explicar un fenómeno. De hecho, la materia ofrecida a examen puede mostrarse fenoménicamente distinta según la naturaleza de las reglas. Es decir, si tenemos,

(7) a. El rey dijo que le trajeran al mensajero

b. "Que me traigan al mensajero", dijo el rey,

podemos fijarnos en las diferencias entre (7a) y (7b), por decir algo, en términos de las propias entre dos órdenes opcionales de

7 Por otra parte, es responsabilidad del "variacionismo" responder, al menos, a estas siete preguntas: (i) ¿Qué tipos de variación lingüística existen?; (ii) ¿cómo están relacionados los diferentes tipos de variación?; (iii) ¿a qué planos del lenguaje y a qué problemas afecta más la variación lingüística y por qué esto es así?; (iv) ¿cuál es la relación entre las investigaciones cuantitativas y las cualitativas?; (v) ¿cuál es la relación de la variación lingüística con la organización de la mente humana?; (vi) ¿cuál es la relación de la variación lingüística con la organización social?; (vii) en suma, ¿qué tiene de valioso el estudio de la variación lingüística para la ciencia del lenguaje? (MARTín Butragueño 1992, pp. 2-24). 
palabras, o en términos de una selección de tipo estilístico, o en términos, si se prefiere, de un fenómeno atribuible a un modelo explicativo de los discursos directos e indirectos. Pero lo que no debe dejar de estar claro es que cualquiera de los tipos de reglas con que podemos observar (7a) y (7b) pertenecen a planos distintos, de modo semejante a como ocurre en (8), al compararlo con (7):

(8) a. El rey ${\text { dijo que } \mathrm{l}_{\mathrm{i}} \text { trajeran al mensajero }}_{\mathrm{i}}$

b. ${ }^{*}$ El rey dijo que $\mathrm{l}_{\mathrm{j}}$ trajeran al mensajero

Parece evidente que no acudiremos a los mismos tipos de reglas para explicar las diferencias entre (7a) y (7b), que para explicar las existentes entre (8a) y (8b).

Imaginemos por un momento que fuera posible suponer varios conjuntos de reglas de índole distinta (sintácticas, pragmáticas o, en otra dimensión, sociales, estilísticas, etc.), de las que pudiera decirse que actúan modularmente sobre diferentes clases de variables. Es decir, que a una variable $\langle y\rangle$ le pueden ser interesantes las reglas de tipo $\mathrm{A}$, pero pueden serle completamente indiferentes las del tipo B. O, de otro modo, tal variable puede ver explicada parte de su distribución por medio de reglas pragmáticas pero no sociales, o sintácticas pero no estilísticas. Podríamos entonces imaginar que a cada variable $\langle y\rangle$ le asignamos una cadena de valores positivos o negativos según le afecten o no los miembros de un catálogo de clases de reglas, esto es,

(9) a. $\langle y>\rightarrow\{|\mathrm{A}|,|\mathrm{B}| \ldots|\mathrm{N}|\}$,

donde las líneas verticales $\|$ indican valor absoluto, que puede manifestarse como [+] o como [-]. Es más, podríamos escribir

b. $<y>\rightarrow\{|\mathrm{A}|,|\mathrm{B}| \ldots|\mathrm{N}|\},\left\{\left|\mathrm{A}^{\prime}\right|,\left|\mathrm{B}^{\prime}\right| \ldots\right.$

$\left.\left|\mathbf{N}^{\prime}\right|\right\}$

donde $|A|,|B| \ldots|N|$ son clases de reglas lingüísticas y $\left|\mathrm{A}^{\prime}\right|, \quad\left|\mathrm{B}^{\prime}\right| \ldots\left|\mathrm{N}^{\prime}\right|$ son clases de variación (histórica, social, etcétera).

Entonces, tendríamos que dedicarnos a dos labores: en primer lugar, a establecer la lista de clases de reglas que pueden afectar a las variables. Por ejemplo, ¿deben las reglas morfológi- 
cas ser incluidas en la lista? ¿Cuántas clases de reglas hay? En segundo lugar, deberíamos tomar una lista lo más amplia posible de variables y asignarles valores en sus cadenas de reglas influyentes, según se propuso en (9). Estas dos operaciones nos permitirían crear una tipología de la variación gramatical. Sabríamos cuál es la repercusión efectiva de las diferentes clases de reglas, y sabríamos cómo agrupar clases de variables, según hubiera o no coincidencias en sus cadenas de variación. Obsérvese que nos estaríamos moviendo con argumentos cuantitativos, en el sentido expuesto en (6). Si todo esto fuera posible, estaríamos en disposición de observar una "radiografía" o modelo de la variación gramatical del español. Serviría ello para entender en qué grado podemos decir que un fenómeno es más variable que otro, o para aceptar o rechazar afirmaciones como las de (10) a (12) - e incluso para saber si es siquiera posible concebir afirmaciones de esa naturaleza:

(10) La categoría de la palabra es poco importante para caracterizar la distribución de las variables morfológicas

(11) Existen semejanzas entre todas las variables basadas en el cambio de modo verbal

(12) Es más probable encontrar variabilidad gramatical en la lista de categorías "simples" $=\{\mathrm{N}, \mathrm{Adj}, \mathrm{V} \ldots\}$ que en las frases $=\{$ FN, FAdj, FV ... $\}$

Vamos a intentar presentar aquí un esbozo, elemental y tentativo, de las clases de reglas que podrían ser consideradas y de la tipología de un grupo de variables del español.

\section{INTENTO DE UNA TIPOLOGÍA}

Examinar un número amplio de variables (cuarenta) tiene dos ventajas demostrativas: 1) poner en tela de juicio la idea preteórica de que los fenómenos variables sólo ocupan un espacio marginal dentro de la gramática; 2) disponer de un repertorio formado por casos dotados de características muy diferenciadas.

Las categorías que se proponen a continuación para organizar la tipología son relativamente simplificadoras. La justificación de ello estriba en tener entre manos -en principio- un 
problema de índole esencialmente empírica y en la carencia actual de una teoría explicativa aceptable. Este planteamiento obliga, en rigor, a hablar más de "ordenación" que de "tipología'".

La selección de las variables ha sido problemática. En primer lugar, se quería partir de los menos apriorismos posibles; de ahí que la búsqueda de variables haya procurado encaminarse a la recolección de fenómenos de todo tipo. Y si se ha querido buscar variedad en los aspectos gramaticales tratados, otro tanto se ha intentado respecto a los factores extralingüísticos: se han incluido variables diacrónicas y sincrónicas, se ha procurado reflejar una amplia geografía (España, México, Venezuela, Chile, Argentina...), con perfiles de variado cuño social y estilístico. Por otra parte, debe tenerse en cuenta una poderosa limitación: la necesidad de encontrar variables que hayan sido estudiadas desde el punto de vista cuantitativo y tomando en consideración, al menos, algunos de estos factores. Sólo cuando se ha adoptado esa perspectiva podíamos, en esta ocasión, interesarnos en establecer un marco adecuado para comparar hechos a veces bastante lejanos, a veces sólo en apariencia lejanos.

En cualquier caso, se han distinguido cuatro clases de variables: morfológicas $(\langle 1 a\rangle$ a $\langle 1 h\rangle)$, categoriales $(<2 a\rangle$ a $\langle 2 k>$ ), funcionales $(<3 a>$ a $<3 l>$ ) y posicionales $(<4 a>$ a $\langle 4 i\rangle)$. Las filas de las matrices de las clases de variables deben leerse así: < var > es el nombre de la variable, [vtes] el catálogo de sus variantes, y las claves de las otras columnas, que podrían ir entre barras verticales, incluyen los factores reguladores de la distribución de las variantes de las variables que se han considerado: $\mathrm{fn}=$ fónico $; \mathrm{mf}=$ morfológico; $\mathrm{st}=$ sintáctico; $\mathrm{sm}=$ semántico; pg = pragmático ocuparían la primera parte de la matriz, y la segunda hs = histórico; $\mathrm{gf}=$ geográfico; se = social; es = estilístico. Empleando esa notación, la regla que afecta a una variable debería leerse como en este ejemplo ${ }^{8}$ :

$$
\underset{\text { variable variantes matriz gramatical matriz variacional }}{\langle 1 b\rangle \rightarrow\left\{\left[\begin{array}{l}
{\left[\mathrm{b}_{1}\right]} \\
{\left[\mathrm{l} \mathrm{b}_{2}\right]}
\end{array}\right], \quad\left[\begin{array}{l}
\mathrm{fn}=-\mathrm{mf}=+ \\
\mathrm{st}=-\mathrm{sm}=- \\
\mathrm{pg}=-
\end{array}\right] \quad\left[\begin{array}{l}
\mathrm{hs}=+\mathrm{gf}=+1 \\
\mathrm{sc}=+\mathrm{es}=+
\end{array}\right]\right.}
$$

${ }^{8}$ Que debería leerse así: dada la variable $\langle\mathrm{lb}\rangle$, la distribución de sus variantes $\left[1 b_{1}\right]$ y $\left[1 b_{2}\right]$ está condicionada por el factor mf en la matriz gramatical y por los factores hs, gf, se y es en la matriz variacional. 
Es discutible (obviamente) qué es lo semántico, qué es lo pragmático, etc.; no vamos a entrar ahora en ello. Aceptamos, convencionalmente, los valores más frecuentes en la bibliografía especializada ${ }^{9}$.

Se han señalado varias condiciones ${ }^{10}$ previas a la defensa de generalizaciones sobre las variables sociolingüísticas. Podemos adaptar así la formulación de algunas de esas condiciones:

Condición 1 sobre las variables sociolingüísticas

Una variable sociolingüística ha de tener al menos una marca " +" en cada una de las dos matrices, gramatical y variacional: $\mathrm{V}\langle\mathrm{x}\rangle$ sociolingüística, $\mathrm{I}$ al menos un "+" en $\mathrm{M}_{\mathrm{G}}$ y en $\mathrm{M}_{\mathrm{V}}$

Esta condición hace referencia al hecho de que no hay razón para otorgar el apellido de "sociolingüística" (u otro parecido) a una variable que no esté asociada a factores en principio extralingüísticos, como el tiempo o el espacio ${ }^{11}$.

(15) Condición 2 sobre las variables sociolingüisticas

a. Estricta: Una variable sociolingüística lo es si tiene -sm y -pg en la matriz gramatical

b. Relajada: Una variable sociolingüística lo es si tiene $-\mathrm{sm}$ en la matriz gramatical

${ }^{9}$ Quizá los tres niveles más problemáticos sean sintáctico/semántico/pragmático. Baste observar que en el primero incluiríamos funciones como \{sujeto, predicado...\}, en el nivel semántico \{agente, paciente...\} y en el pragmático \{tema, rema...\} (aunque sea discutible la relación entre lo informativo y lo pragmático; por lo demás, es obvio que estos niveles no se agotan en esos listados). A nosotros nos han sido especialmente útiles Hernanz y Brucart 1987, Riemsdijk y Williams 1990, Levinson 1983, Escandell Vidal 1993, entre otros. Puede resultar también discutible la asignación de algunas variables a ciertos tipos; por ejemplo, hemos preferido dar al modo un valor categorial.

${ }^{10}$ Así, recuérdese lo establecido por Labov 1972, p. 271 -cfi. supra n. 1-, y también observaciones de LAVANDERA como la de que "preferiría no analizar como "variables sociolingüísticas" formas sintácticas alternantes al menos que se den otras dos condiciones: (1) que pueda probarse que son las portadoras de significación social y estilística... y (2) que pueda probarse que son un tipo de instrumento del lenguaje semejante a las variables fonológicas, es decir, elementos cuya propiedad definitoria es una covariación cuantificable y con respecto a los cuales las relaciones de frecuencia mismas son las que señalan diferencias"' (1984, pp. 45-46), entre otras.

11 Véase, por otra parte, LAVANDERA 1984, p. 44. 
La versión estricta de la Condición 2 exige la inmutabilidad del significado semántico y pragmático al alternar las variantes para poder hablar, en rigor, de variable. Esto, como se verá un poco más abajo, reduce muy notablemente el catálogo de fenómenos candidatos a variables sociolingüísticas. La versión relajada reduce también sensiblemente el catálogo e incluye prácticamente clases enteras de variables, como las posicionales.

Es fácil ver que estas condiciones descansan en un supuesto más general, que podríamos describir así:

(16) Hipótesis 1

Una variable lo es si tiene, al menos, una marca " + " en una cualquiera de las dos matrices, gramatical o variacional. Es decir: $\forall<x>$, $\exists$ al menos un " +" en $M_{G}$ o en $\mathrm{Mv}_{\mathrm{v}}$

A veces (como en Delbecque 1990, 1991, 1992), se ha asumido y explotado el papel del significado como, podríamos decir, constructor de contextos, y se ha subrayado la importancia de la existencia de variación en sí misma, sin imponer condiciones sobre los factores de influencia. Esto supone desplazar el interés variacionista de la variable sociolingüística a la variable a secas ${ }^{12}$; es decir, se eliminan las Condiciones 1 y 2 y se eleva la Hipótesis 1 a categoría de condición:

$$
\begin{aligned}
& \text { Condición } 3 \text { sobre las variables } \\
& \mathrm{V}\langle\mathrm{x}\rangle \text {, } \mathrm{G} \text { al menos un "+ " en } \mathrm{M}_{\mathrm{G}} \text { o en } \mathrm{M}_{\mathrm{V}}
\end{aligned}
$$

Como puede observarse, desaparece la Condición 2 y cambia el operador de la Condición 1, que era " $y$ ", mientras que en la Condición 3 es "o".

Podemos, por último, y a la luz de lo comentado al considerar la escala de observación de las variables, imponer una condición sobre la asignación de marcas " + " o " — " a los factores reguladores de las variables:

(18) Condición 4 sobre las marcas en las reglas de cualquier clase de variable

12 Esta posibilidad ha sido criticada, especialmente pensando en los casos en que los factores extralingüísticos no parecen desempeñar ningún papel (cf. LaVANDERA 1984, pp. 37-46). 
$\mathrm{El}$ argumento decisivo para fijar la marca debe ser el cuantitativo, en el sentido de (6)

Esta Condición asegura, en realidad, que se esté empleando un enfoque variacionista y, en términos prácticos, ayuda a verificar la metodología empleada en los análisis.

Este conjunto de condiciones nos proporciona algunas herramientas para desbrozar los cuadros de variables y nos ofrece preámbulo para intentar luego algunas generalizaciones sobre la tipología.

En los cuadros que siguen, las marcas " + " indican la acción pertinente de alguna clase de reglas; la " - " niega la acción; "?", vale para los casos dudosos y, muchas veces, para la falta de datos adecuados. Las marcas entre paréntesis necesitan ser aclaradas en cada ocasión. Suelen indicar que la marca es como aparece, pero que conviene restringir o excluir de la variable algún subconjunto de datos para que tenga una significación adecuada.

\section{Cuadro 1}

Variables de tipo morfológico

\begin{tabular}{|c|c|c|c|c|c|c|c|c|c|c|c|}
\hline & $<$ var $>$ & [vtes] & $f_{n}$ & $m f$ & st & $s m$ & $p g$ & $h s$ & $g f$ & se & es \\
\hline$\langle a\rangle$ & verbo aux. & ser/haber & - & + & - & - & - & $?$ & + & + & + \\
\hline$\langle b\rangle$ & terminación verbal & $-m o s /-n o s$ & - & + & - & - & - & + & + & + & + \\
\hline$\langle c\rangle$ & terminación verbal & -ste/-stes & - & + & - & - & - & + & + & + & + \\
\hline$\langle d\rangle$ & terminación verbal & $-r a /-s e$ & - & + & + & - & - & + & + & + & ? \\
\hline$\langle e\rangle$ & terminación verbal & -ra/-ría & - & + & - & - & - & + & + & + & ? \\
\hline$\langle f\rangle$ & $\begin{array}{l}\text { expresión del } \\
\text { futuro }\end{array}$ & $\begin{array}{l}\text { cantarél } \\
\text { voy a cantar }\end{array}$ & - & + & - & $(-)$ & - & + & + & + & + \\
\hline$\langle g\rangle$ & $\begin{array}{l}l e, \text { la y } l o \text { en } \\
\text { Madrid }\end{array}$ & func./ref. & - & + & - & - & - & + & + & - & ? \\
\hline$\langle h\rangle$ & $\begin{array}{l}\text { le, la y lo en } \\
\text { Castilla la Vieja }\end{array}$ & func./ref. & - & + & - & - & - & + & + & + & ? \\
\hline
\end{tabular}

Saltan a la vista varias regularidades en el cuadro de las variables que hemos llamado morfológicas. En primer lugar, es constante la asignación + mf; de hecho, esa marca puede servirnos provisionalmente para definir el tipo I de variables:

(19) Tipologia 1 (provisional)

Las variables de tipo I se caracterizan por mostrar $+\mathrm{mf}$ 
Otra circunstancia bastante evidente es la abundancia de marcas " - " en la matriz gramatical, frente a la abundancia de marcas “ + " en la matriz variacional, lo que puede apreciarse incluso visualmente. La regla típica asociada a las variables de tipo I es como la correspondiente a la regla $\langle b\rangle$ :

$$
<\text { term. verbal }>\rightarrow\{[- \text { mos }],[-n o s]\},\left[\begin{array}{ll}
-\mathrm{fn} & +\mathrm{mf} \\
-\mathrm{st} & -\mathrm{sm} \\
-\mathrm{pg} &
\end{array}\right]\left[\begin{array}{l}
+\mathrm{hs}+\mathrm{gf} \\
+\mathrm{sc}+\mathrm{es}
\end{array}\right]
$$

Esta regla describe casos como el de este ejemplo:

(21) Entonces mi papá nos llamó para que fuéranos a buscar un ganado para allá para el carrizo.

(Navarro Correa 1992, p. 99)

En efecto, a juzgar por lo que parece mostrar Navarro Correa con datos de Valencia, Venezuela, no parece que el cambio de -nos por -mos tenga mayores efectos sintácticos, semánticos o pragmáticos, mientras que parece haber argumentos razonables para asignar " +" a todos los partícipes de la matriz variacional $^{13}$. No hay problema en asignar -fn, pues si los casos quedan restringidos a formas esdrújulas, basta con incluir la restricción en la definición de la variable ${ }^{14}$.

Contornos parecidos tienen otras muchas variables, como $\langle c\rangle$ :

${ }^{13}$ Con todo, debe tomarse en cuenta que Navarro Correa (1992) se ocupa más del grado de relevancia y su condicionamiento social que de los condicionantes lingǘsticos: se trabajó con 484 informantes estratificados por sexo, edad, escolaridad e ingresos; el material se complementó con una encuesta escrita (+es): "se observa que se reducen significativamente los índices de frecuencia de las formas estigmatizadas. Tal fenómeno era esperable, dada la diferencia de formalidad existente entre la expresión oral y el registro escrito" (p. 103). Valgan estos comentarios para todas las variables descritas a partir de las observaciones de Navarro Correa (1992).

${ }^{14}$ Aunque Navarro Correa anota que esto "permite suponer que la posición de -mos respecto a la sílaba acentuada contribuye a favorecer el cambio" (1992, p. 99). Por otra parte, no es seguro que el procedimiento de concreción de contextos esté plenamente justificado (véase LAVANDERA 1984, pp. 44-45). 
(22) a. No sé si fuiste por fin a ver a tu tío

b. No sé si fuistes por fin a ver a tu tío

Uno de los problemas es establecer dónde termina la variable fónica y empieza la morfológica; algunos han llamado a este terreno intermedio variable morfonológica. Al marcar $+\mathrm{mf}$ y -fn lo que se quiere decir no es tanto que en el fenómeno nada hay de fónico y sí de morfológico, como expresar el hecho de que ciertos factores de índole morfológica (por ejemplo, la analogía) son más propicios para explicar la distribución de las variantes que otros de índole puramente fónica.

En general, no parece haber muchas diferencias entre las variables de la esfera verbal, $\langle a\rangle$ a $\langle f\rangle$ y las de la esfera nominal, $\langle g\rangle$ y $\langle h\rangle$. Esto nos permite postular una primera generalización:

\section{Generalización 1}

En las variables del tipo I hay pocas diferencias entre las que afectan a la esfera nominal y las que afectan a la esfera verbal

Quiere esto decir que no se esperan demasiadas diferencias de comportamiento entre dos variables que estén actuando sobre dos categorías diferentes; la sugerencia parece demasiado arriesgada como para intentar aplicarla sobre variables no morfológicas.

El hecho ya comentado del mayor número de marcas " +" en la segunda parte de la tabla nos permite formular la

\section{Generalización 2}

Las variables del tipo I tienden a cubrir con " + " todas las secciones de la matriz variacional

El problema en ciertos casos es la falta de datos o la duda ante ellos. Por ejemplo, la variable $\langle a\rangle$, que corresponde a ejemplos como éste:

(25) Entonces se fue y no volvió más [...] Me fuera casado con él, me fuera mejor (Navarro Correa 1992, p. 99)

Se ha marcado con ?hs, aunque debe haber una asociación susceptible de describirse históricamente y, en consecuencia, podría 
haberse marcado con " + ". Precisamente, la columna $|\mathrm{hs}|$ presenta el problema de su campo de acción. Si se adopta una perspectiva muy amplia, casi no habría variable que no deba llevar " + "; otra posibilidad es restringir su naturaleza a los cambios en curso. Otros casos con problemas semejantes son las variables $\langle e\rangle$ y $\langle k\rangle$, ambas ?es; $\langle e\rangle$ se refiere a la alternancia entre cantara y cantaría en la apódosis de las condicionales también en Valencia, Venezuela (Navarro Correa 1989) ${ }^{15}$. Como se verá más adelante, el verdadero problema con |es| se presenta cuando en la matriz gramatical existen marcas como + pg; cabe entonces entender el estilo de dos maneras: como correlación según algún parámetro (por ejemplo el de formalidad), o como exponente de la construcción del significado. El problema es delicado y se comentará un poco más abajo.

La variable $\langle f\rangle$, expresión del futuro, se ha marcado como (-)sm por considerar que las formas sintéticas y analíticas son neutralizables en algunos contextos:

\section{(26) a. Voy a ir a Madrid}

b. Iré a Madrid

De ser correcto el planteamiento ${ }^{16}$, tenemos dos opciones: ocu-

15 "La sustitución de la vieja forma cantara por cantaría en la apódosis es hoy un hecho consumado tanto en la expresión oral como escrita del español peninsular; pero no sucede lo mismo en algunas regiones americanas" (NAVARRo Correa 1989, p. 121): +hs, +gf; Navarro ofrece datos en los que, "en la apódosis. . cantara conserva todavía gran vitalidad frente a la variante mayoritaria cantaría, sobre todo, entre los sectores de más edad, menos ingresos y escolaridad más baja"' (p. 123): +sc. Por otra parte, sería interesante disponer de datos suficientes como para saber con claridad si se trata simplemente de una variable morfológica, o bien de una variable en que pueda implicarse el cambio de modo; en este último caso, debería figurar junto a las presentadas en $\langle 2 \mathrm{a}\rangle \mathrm{a}\langle 2 \mathrm{~d}\rangle$.

16 "El factor semántico decisivo en el uso de ambos futuros es, a nuestro juicio, la "conexión" de la acción verbal con el acto de habla. Esta conexión - sea temporal, espacial, situacional- puede ser positiva o negativa. En el primer caso se prefiere el futuro analítico; en el segundo, el futuro sintético" (BERSCHIN 1987, p. 104). Para ths, +gf y +es, se recuerda que "(1) el uso del futuro analítico, que aparece por primera vez en el siglo XvI, ha ido aumentando hasta la actualidad. (2) Existe, en el español actual, una variación regional y estilística de ambos futuros: el futuro analítico se emplea más en el español americano que en el español peninsular; desde el punto de vista estilístico o diafásico, el futuro sintético predomina en la lengua escrita, elaborada, mientras la lengua hablada y espontánea favorece el futuro analítico" 
parnos sólo de los casos intercambiables (si es que los hay), e introducir una notación semejante a la otorgada, o bien dar " +" a sm o a pg, e introducir el mismo tipo de problemas que se han de ver con otras clases de variables.

A la vista de los datos de esta primera tabla, nos sentiríamos tentados a preguntarnos si, dada una marca " + " en uno de los puntos de la matriz variacional, puede esperarse que aparezca " + " en los puntos superiores. De tener esto algún fundamento, podría formularse la siguiente hipótesis general ${ }^{17}$ :

\section{Hipótesis 2}

$\mathrm{Si}$ un $\mathrm{Y}_{\mathrm{n}}$ de la matriz variacional está asociado a " + ", esto implica que $\mathrm{Y}_{1}$ a $\mathrm{Y}_{\mathrm{n}-1}$ están asociados a "+". Esto es, si un $\left|Y_{n}\right| \in M_{V} \rightarrow$ "+", entonces $Y_{1}$ a $Y_{n-I} \rightarrow$ "t"

Esta hipótesis presenta diversos problemas, pero probablemente tiene validez parcial, como se verá más abajo. Para el caso del tipo I, sin embargo, vamos a proponer elevarla a categoría de generalización:

$$
\begin{aligned}
& \text { Generalización } 3 \\
& \text { En el tipo } \mathrm{I} \text {, si un }\left\{\mathrm{Y}_{\mathrm{n}} \mid \in \mathrm{M}_{\mathrm{V}} \rightarrow \text { "“+", entonces } \mathrm{Y}_{1}\right. \text { a } \\
& \mathrm{Y}_{\mathrm{n}-\mathrm{I}} \rightarrow \text { a }+,
\end{aligned}
$$

En cuanto a $\langle g\rangle$ y a $\langle h\rangle$, se trata del uso funcional o referencial de $l e, l a, l o$ en dos lugares diferentes, Madrid (Moreno $e t$ al. 1988) ${ }^{18}$ y Castilla la Vieja (Klein 1979). Resulta interesante la oposición en las últimas marcas de la matriz variacional -dejemos de lado ?es en ambas: - se para Madrid y + sc para Castilla la Vieja. Obsérvese que no hay ningún problema con respecto a la Generalización 3.

La variable $\langle d\rangle,-s e /-r a$ en contextos donde son sintácticamente equivalentes, presenta la interesante peculiaridad de ser

\footnotetext{
(p. 101).

17 Véase también Martín Butragueño 1992, pp. 7-11.

18 Respecto a los elementos de la matriz variacional: "El sexo no se presenta como un factor determinante en la aparición de los pronombres $l e$, la, lo en sus usos estimilógicos o no etimológicos" (MORENO et al. 1988, pp. 106107); "no son fenómenos que se originan en la variable sociológica "edad"" (p. 109); "en líneas generales, nos encontramos en disposición de afirmar que estos fenómenos no obedecen a variables de naturaleza sociológica («edad", "sexo») ni geográfica" (p. 109). El estilo no se consideró.
} 
+st; se debe esa marca a que, al parecer, al menos en algunas circunstancias, la prótasis de las condicionales favorece un poco más la aparición de formas en -se (Navarro Correa 1989) ${ }^{19}$.

Si leemos la matriz de la clase morfológica considerando las condiciones formuladas un poco más arriba, todas esas variables las cumplen sin ningún problema. En efecto, todas tienen al menos una marca " + " en la matriz gramatical; y también al menos una marca " + " en la matriz variacional (Condición 1). Cumplen la Condición 2 estrictamente, pues todas, en principio, pueden ser -sm y -pg; cumplen, obviamente, la Condición 3 y en todas el argumento cuantitativo ha sido trascendente (Condición 4). Puede decirse que son variables óptimas para cualquier tratamiento sociolingüístico, etc. A eso se debe, tal vez, que muchas de ellas aparezcan como preocupación ya incluso en trabajos tradicionales.

Cuadro 2

Variables de tipo categorial

\begin{tabular}{|c|c|c|c|c|c|c|c|c|c|c|}
\hline & $<$ var $>$ & {$[v t e s]$} & $f n m f$ & st & $s m t$ & $\operatorname{sg}$ & $h s$ & $g f$ & se & es \\
\hline$a>$ & $\begin{array}{l}\text { subjuntivo e infini- } \\
\text { tivo con para }\end{array}$ & nf. & -+ & + & + & + & ? & $?$ & ? & ? \\
\hline$\langle b\rangle$ & $\begin{array}{l}\text { indicativo y sub- } \\
\text { juntivo en la próta- } \\
\text { sis, Buenos Aires }\end{array}$ & ind./subj. & -+ & + & & + & ? & + & + & ? \\
\hline$\langle c\rangle$ & $\begin{array}{l}\text { cambio de modo, } \\
\text { Buenos Aires }\end{array}$ & ind./subj. & -+ & + & + & + & - & - & - & + \\
\hline$\langle d\rangle$ & $\begin{array}{l}\text { indicativo y } \\
\text { subjuntivo en } \\
\text { Covarrubias }\end{array}$ & ind./subj. & -+ & + & + & + & $?$ & + & ? & ? \\
\hline$\langle e\rangle$ & relativo & $\begin{array}{l}q u e \\
\text { izador }\end{array}$ & -+ & $+)$ & - & - & $?$ & - & - & $?$ \\
\hline$\langle f\rangle$ & $\begin{array}{l}\text { recategorización } \\
\text { del adj. en adv. }\end{array}$ & $\begin{array}{l}\text { adj./adj. } \\
\text { advdo. }\end{array}$ & -- & + & - & - & + & + & ? & ? \\
\hline$\langle g\rangle$ & $\begin{array}{l}\text { generalización del } \\
\text { art. }\end{array}$ & $\operatorname{art} . / \emptyset$ & -- & + & - & - & + & $(+)$ & $(+)$ & $(+)$ \\
\hline$\langle h\rangle$ & $\begin{array}{l}\text { tipo de determi- } \\
\text { nante }\end{array}$ & $\begin{array}{l}\text { art. } / \text { dem. } . / \\
\text { cuant. } / . .\end{array}$ & -- & + & + & + & - & - & - & + \\
\hline$\langle i\rangle$ & secuencia de preps. & por/a por & -- & + & - & - & + & + & + & ? \\
\hline
\end{tabular}

19 Además, "es bien conocido el progresivo desuso de la variante en -se en ambos pretéritos de subjuntivo" (NAVArro Correa 1989, p. 117): esto justificaría + hs; existen diferencias geográficas $(+g f)$, por ejemplo entre Sevilla y México, etcétera. 


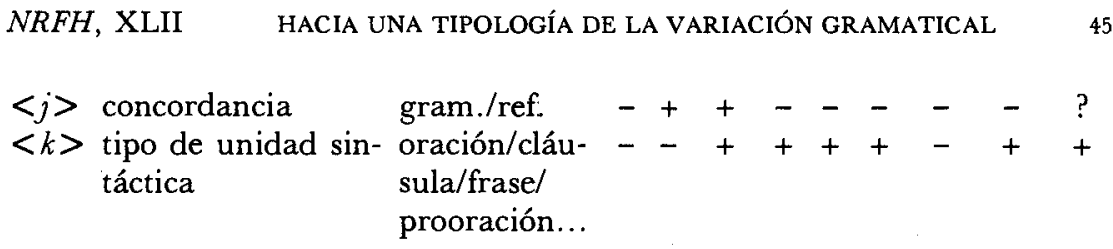

La lectura del cuadro de variables de tipo categorial es algo más compleja, pero también es posible encontrar algunas regularidades. Al decir "categorial" nos referimos a clases de palabras, de frases, de oraciones.

Al igual que en el cuadro anterior, y como ocurrirá también en el siguiente, todas las variables van marcadas como $-\mathrm{fn}$. Y todas reciben, en principio, la señal + st. Varias presentan $+\mathrm{sm}$ o + pg, y eso hace discutible su carácter de variables. Por fin, y a diferencia del cuadro anterior, los signos " + " son mucho menos abundantes en la matriz variacional, lo que también es sintomático.

Uno de los casos más sencillos de explicar es el de la variable $\langle i\rangle$, representativa de ejemplos como éste:

(29) a. Voy por agua

b. Voy a por agua

En efecto, no parece que sea necesario acudir a factores fónicos ni morfológicos, ni tampoco a explicaciones semánticas o pragmáticas; quizá baste pensar en la insurgencia de la preposición $a$, compañera frecuente de $i r$. Cumple esta variable todas las condiciones propuestas, y respeta también las hipótesis 1 y 2 .

Otra variable regulada de modo semejante a ésta es la $\langle g\rangle$, que da cuenta del proceso de generalización del artículo en español:

(30) a. med.: tovieron castellanos el puerto bien guardado

b. act.: tuvieron los castellanos (Company 1991, p. 83)

La marca -sm para esta variable debe discutirse un poco. En efecto, se ha postulado que la categoría 'artículo' debió extenderse inicialmente ante sustantivos concretos y tangibles (Company 1991, pp. 103-104): primero, con sustantivos genérico-humanos sujeto (p. 101), después, la presencia fue potenciada por el sujeto (p. 102) y, en tercera etapa, el artículo progresó a sustantivos abstractos y de masa (p. 102). Ahora bien, el problema con cualquier variable histórica es el grado de convivencia de ambas for- 
mas (esto es, castellanos/los castellanos) y el grado de diferenciación semántica y pragmática de dos formas contemporáneas ${ }^{20}$. Es decir, si en un momento dado el artículo se asoció a los sustantivos animados y a la función sujeto ${ }^{21}$, la pregunta es: el empleo del artículo en esa época, ¿inducía a interpretar a un sustantivo como animado, aunque fuera figuradamente?, ¿colaboraba su empleo a "representar" la función sujeto? Si la respuesta fuera afirmativa, entonces deberíamos marcar $+\mathrm{smo}+\mathrm{pg}^{22}$. En cuanto a la matriz variacional, se hace difícil pensar en un cambio de este tipo sin dar tiempo a una extensión geográfica, social y estilística. Por eso se ha marcado $(+)$ en las tres últimas casillas.

Otras dos variables que presentan un patrón semejante al que se está comentando, son la $\langle f\rangle$ y la $\langle k\rangle$. La $\langle f\rangle$ o recategorización del adjetivo en adverbio podría ejemplificarse así:

(31) a. Sabe feo

b. Es feo

Entre las consideraciones que cabe hacerse está si el feo de (31a) es el mismo que el de (31b). Otra cuestión es si no sería mejor leer esta supuesta variable como funcional, más que como categorial. En cualquier caso, parece poseer una interesante matriz variacional.

${ }^{20}$ Resultan muy interesantes estas observaciones: "Los diferentes copistas eran sensibles a la presencia o no del artículo e introdujeron el mismo en frases sustantivas que carecían de él"' (Company 1991, p. 83); "Las modificaciones que con respecto al artículo hicieron los copistas no se llevaron a cabo en todas las épocas sobre la misma clase de sustantivos, ni en una misma distribución o función de esas voces, lo cual apunta hacia un lento y gradual cambio sintáctico en el comportamiento del artículo y sustantivo" (p. 84).

${ }^{21}$ Lo que es comprensible, pues "los sintagmas nominales que designan seres animados suelen estar más cualificados en muchas lenguas para actuar en el núcleo predicativo como sujetos y en la periferia, como objetos indirectos; por su parte, los sintagmas que denotan seres inanimados suelen funcionar en las lenguas como objetos directos en la predicación y como complementos circunstanciales en la periferia. Denominamos este fenómeno "Ley de la Preeminencia de la Animación" (LPA)" (MORENo CABRERA 1987, p. 48). Prueba de la importancia de esta ley es que más de una de las variables aquí consideradas encuentra en ella su marco explicativo.

${ }^{22}$ Es posible, en cambio, marcar " - " si se considera que las características semánticas estaban ya implícitas en los sustantivos: el artículo "treparía" de un modo predeterminado y, en esencia, no habría cambio de significado. 
Más problemático nos parece el caso de $\langle k\rangle$, el tipo de unidad sintáctica: oración, cláusula, frase ..., tal como se encuentra formulado en Lope Blanch (1987) ${ }^{23}$. Se ha asignado + sm y + pg, aunque podríamos hacer abstracción de esos factores y emplear la variable para medir la complejidad de un texto (entendida ahora como el índice resultante de clasificar los tipos variantes). Habría todo un conjunto de reglas más discursivas que gramaticales, en sentido estricto, y quizá ésta sea una de ellas. Este hecho nos sugiere una generalización tentativa, que debería formularse en términos parecidos a éstos:

\section{Generalización 4}

Es más probable que una variable tenga repercusiones discursivas cuanto más altas sean las categorías a las que afecta

Por "discursivo" quiere decirse 'más allá del límite oracional'. "Altas" significa que una oración lo es más que una frase, y ésta más que una palabra. Por provisional que sea, esta generalización refleja, en parte, la importancia del contexto. Cuanto más complejas sean las categorías puestas en juego, más son las posibilidades de elección y de ejercer significaciones diferenciadas ${ }^{24}$. Este "control" del contexto puede moverse en la linde oracional, pero también fuera de ella, y por eso este tipo de variables son mejores candidatas a la tipificación de discursos que de oraciones. Ade-

23 " Se desprende que los conceptos de oración, período (y oración compuesta), frase y prooración pertenecen a un nivel diferente del que corresponde a la cláusula. Los primeros se excluyen entre sí, pero ninguno de ellos es excluyeme de la cláusula. Una oración, en efecto, no puede ser a la vez frase o período, ni éste puede ser prooración o frase, etc. Pero cualquiera de ellos puede constituir una cláusula, siempre que aparezca como expresión autónoma del discurso"' (LOPE BLANCH 1987, pp. 14-15). Ciertas variantes estarían asociadas a "géneros": "la frase resulta ser característica de la expresión poética, en tanto que las prooraciones son formas casi exclusivas del diálogo" (p. 63), lo que justifica +es; además, la cláusula popular es más breve y más simple que la culta, las oraciones cultas son más complejas, la yuxtaposición y la coordinación son más comunes en habla popular que en culta, la estructura típica de la cláusula popular suele ser muy sencilla, son más abundantes las relaciones nexuales entre cláusulas sucesivas en el habla popular que en la culta, algunas formas de relación sintáctica son poco usuales entre incultos, no hay un divorcio completo entre uno y otro nivel $(+\mathrm{sc})$. En cuanto a ths, "la estructura sintáctica de la prosa castellana se fue enriqueciendo, a partir de su desnuda sencillez primitiva, hasta llegar, en poco más de dos siglos, a esquemas sumamente complejos y variados"' (p. 169).

\footnotetext{
${ }^{24}$ Cf. n. 4.
} 
más, lo que cabe esperar de las variables afectadas por la Generalización 4 es que sean $+\mathrm{sm}$, + pg.

La construcción del contexto es también lo que explica las marcas + sm y +pg en la variable $\langle h\rangle$, tipo de determinante ${ }^{25}$. Si tomamos

(33) a. $E l$ hombre estaba en la estación

b. Un hombre estaba en la estación

c. Aquel hombre estaba en la estación

d. Ese hombre estaba en la estación

e. Cierto hombre estaba en la estación

El determinante nos sirve para construir el contexto. Si esta oración comenzara una narración, el empleo de los diferentes determinantes exigiría al lector la buena fe de construir contextos adecuados. Existen, por lo demás, limitaciones a esta libertad contextual. Una de ellas puede ser la doble determinación:

(34) a. ¿Te acuerdas del hombre que vimos en la estación?

b. ¿Te acuerdas de un hombre que vimos en la estación?

c. ¿Te acuerdas de aquel hombre que vimos en la estación?

d. ¿Te acuerdas de ese hombre que vimos en la estación?

e. ¿Te acuerdas de cierto hombre que vimos en la estación?

donde que vimos en la estación tiene ya papel acotador. Por cierto, que es incluso posible establecer una generalización provisional:

(35) Generalización 5

La proximidad de dos casos de la misma variable limita las posibilidades contextuales

Quizá la implicación más interesante de esta generalización es recordar cómo, en el juego de las elecciones lingüísticas, puede ser muy importante para el hablante lo que dijo hace un momento (o lo que está a punto de decir), bien para emplear la variatio, bien para servirse de paralelismos.

Antes de examinar el problema del estilo, consideremos las variables $\langle e\rangle$ y $\langle j\rangle$, con estructuras en las que abundan los signos " - ". Al hablar de pronombres relativos, debe observarse lo siguiente: la variable entendida como 'qué pronombre se ha

${ }^{25}$ Muy útil es Leonettí Jungl 1990; véase también Moreno Cabrera 1987, pp. 15-29 y García Fajardo 1989. 
usado' encierra el problema de no intercambiabilidad de todos los pronombres en todos los contextos; de hecho, pueden construirse índices textuales generalizadores, por ejemplo oponiendo que a no que (la suma de todos los demás). Otra posibilidad es concretar la variable, por ejemplo considerando la alternancia entre quien y el que generalizadores, o entre el qué interrogativo y lo que en ciertas posiciones (por eso hemos anotado $(+) \mathrm{st})^{26}$ :

(36) a. Quien quiera que venga

b. El que quiera que venga

c. Sabes qué te digo

d. Sabes lo que te digo

Al igual que esta variable, la $\langle j\rangle$ presenta una precaria matriz variacional:

(37) a. Gran número de personas asistió al evento

b. Gran número de personas asistieron al evento

En (37a) el verbo concuerda con número, que se interpreta como núcleo de gran número de personas; en (37b), en cambio, se otorga concordancia plural, pues se interpreta como núcleo a personas, y a gran número de como cuantificador. El interés de construir las matrices de variación es, precisamente, el de poder comparar cómo una variable varía máś intrínsecamente que otras, o menos, aunque por el momento sólo estemos en disposición de explicar algunos de los fenómenos.

Un hecho insistente y problemático es la aparición de marcas " + " o "?" ante $\mid$ es $\mid$, incluso cuando las otras casillas de la matriz variacional estén marcadas negativamente; esto parecería ir contra la Generalización 3 , si queremos extenderla más allá del tipo I, pues esa generalización favorece las marcas " + " a la izquierda, en la matriz variacional. Deberíamos, y no es fácil, distinguir dos clases de $|e s|^{27}$ :

(38) a. El estilo en cuanto correlación de otra medida

b. El estilo en cuanto elección para construir el significado

${ }^{26}$ García Mouton, Martín Butragueño y Molina Martos 1991 emplearon datos procedentes de la norma culta de seis ciudades: Buenos Aires, México, Bogotá, Caracas, Madrid y Sevilla.

${ }^{27}$ Sobre el estilo en sociolingüística, cf. MORENo 1992. 
Por ejemplo, una persona puede aspirar la $-s$ implosiva en un estilo de habla relajado (sentido 38a), pero también puede aspirarla para imitar a una persona de otro lugar (sentido 38b) - expresada la distinción en términos elementales. Si tomamos |es| en el sentido (38a), buena parte de las marcas asignadas serían, probablemente, "-". Si lo tomamos en el sentido de (38b), prácticamente todas las variables llevarían asociado el " + ". De ahí la ambigüedad de muchas de las asignaciones realizadas. Por ejemplo, la variable $\langle h\rangle$, tipo de determinante, recibiría, con claridad, " + " en el sentido (38b), pero probablemente " - " en la interpretación (38a). Debemos entonces reformular la Generalización 3 del siguiente modo:

Generalización 6 (3 revisada)

Si un $\left|Y_{n}\right| \in M_{V} \rightarrow$ " +", entonces $Y_{1}$ a $Y_{n-1} \rightarrow$ " +", tomando en cuenta que |es| debe interpretarse como correlación de otra medida

Esta restricción permite "salvar" para mayor número de casos la generalización. Ése era uno de los problemas que se planteaban con las cuatro primeras variables que aparecían en el cuadro, todas ellas variantes de una misma variable general, el < cambio de modo $>$. Las dos columnas críticas son $|\mathrm{sm}|$ y $|\mathrm{pg}|$, y en ellas concentraremos nuestra atención.

Estas variables se refieren a los siguientes casos:

(40) Variable $\langle a\rangle$

a. para inme allí

b. para que ella viera las condiciones (Morales 1989, p. 30) ${ }^{28}$

(41) Variable $\langle b\rangle$

a. Si él tiene un disgusto, no viene y me dice: Mirá, me pasó esto y esto. Nada: se lo calla, se lo traga

b. Si pudiese volver el tiempo atrás, me preocuparía más en eso

c. Si tendría que hacer una cosa como esa, me gustaría

(Lavandera 1984, pp. 24-25)

${ }^{28}$ Las construcciones con para se deben caracterizar atendiendo a tres factores fundamentales: "Las estructuras que forman; los enlaces referenciaIes que se establecen entre los argumentos de la subordinada y matriz; la mayor o menor marca temporal que manifiesta el verbo subordinado" (MoRAles 1989, p. 41). 
(42) Variable $\langle c\rangle$

a. Mientras que a vos no te falte nada

b. Mirá, Gerardo, ¿a vos te falta algo?

(Lavandera 1990 , p. 336$)^{29}$

(43) Variable $\langle d\rangle$

a. Es como si quedaría muerto, ¿no?, pero no importa porque ya no me vuelve a molestar

b. Es como si te lo quisieras llevar todo para ti, ¿no?, lo quisieras llevar a pedacitos (Silva-Corvalán 1989, p. 132) ${ }^{30}$.

La complejidad de estas variables parece favorecer la interpretación $+\mathrm{sm},+$ pg. Así, en el caso de $\langle d\rangle$, parecen existir diferencias de significado entre, por ejemplo, las variantes $-b a$ y -ría en la apódosis de las condicionales irreales de no pasado, lo que justificaría +sm; además, al menos en algunos casos, "la ambigüedad... se resuelve en el contexto de la comunicación, que generalmente permite al interlocutor recuperar el significado apropiado" (Silva-Corvalán 1989, p. 137), observación que parece apoyar + pg. También debe asignarse $+\mathrm{sm} a\langle b\rangle$, pues se postuló que los tiempos verbales están categorizando "la probabilidad que tiene una situación hipotética de convertirse en un acontecimiento real"' (Lavandera 1984, p. 24), y se asignaron las

${ }^{29}$ Muy interesantes las observaciones de LAVANDERA acerca del papel del estilo: "Mi hipótesis sostiene que, por ejemplo, las formas "causativas" no sólo aparecen en términos de "causa" y "efecto" sino que se las puede referir a las pasivas, por ejemplo, en textos destinados a crear una situación más informal. Obsérvese que, también en mi opinión, el estilo de los textos es uno de los medios empleados para establecer algunas de las características de las situaciones, como formalidad o informalidad, en lugar de que las situaciones constituyan factores que determinan el estilo del habla" (1990, pp. 354-355). Este trabajo de Lavandera, inicialmente publicado en 1983, se encuentra también recogido en su libro de 1984, pp. 125-147.

${ }^{30}$ En Covarrubias, "el condicional no sólo ocurre en la prótasis de oraciones condicionales, sino que en todo contexto en el que otras variedades del español sólo aceptan el imperfecto de subjuntivo" (SILva-CoRvalán 1989, p. 132). Se estudian tres contextos: $a$ ) oraciones condicionales, de las que son ahora interesantes las irreales de no-pasado y las irreales de pasado; en las primeras, -ralse, -ría y (-ba) están asociadas en la prótasis a +contrario a la realidad y a \pm probable en el futuro, en las apódosis, en cambio, -ba se asocia a +contrario a la realidad y a +[mayor probabilidad], y -ría a +contrario a la realidad y a +[menor probabilidad]. En las irreales de pasado, "la prótasis de las oraciones condicionales es el único contexto de pasado en el que el condicional parece haber empezado a sustituir al subjuntivo en Covarrubias" (p. 136); $b$ ) oraciones subordinadas adverbiales y adjetivales y $c$ ) oraciones subordinadas nominales. 
categorías de [real] -ejemplo (41a)-, [contrario] - (41b)-o [posi$b l e]-(41 \mathrm{c})-{ }^{31}$; en cuanto a + pg, Lavandera señala que para clasificar los ejemplos utilizó diferentes clases de información: a veces los items lexicales, a veces el tiempo de la apódosis, a veces, y debería subrayarse, "la decisión de clasificar un contexto en un grupo u otro depende de información que el hablante ofreció en una parte anterior de su discurso" (1984, p. 25). Con un criterio semejante, podemos asignar $+\mathrm{sm}$ a $\langle c\rangle$, en tanto que la modalidad marcada morfológicamente indica \pm aserción, y + pg, por la importancia del contexto en la selección del modo y por la contribución del cambio de modo en la construcción del significado textual. Observaciones semejantes valen para $\langle a\rangle$; también en la elección de infinitivo o subjuntivo parece haber buenas razones para asignar $+\mathrm{sm}$ y probablemente $+\mathrm{pg}^{32}$.

A la vista de todos estos hechos, podríamos ampliar y revisar la tipología ya propuesta:

(44) Tipología 2 (provisional)
a. Tipo I: $+\mathrm{mf}+\mathrm{hs}+\mathrm{gf}+\mathrm{sc}$
b. Tipo II: + st

Esta tipología, aparte de su valor distintivo, intenta reflejar tamkién el hecho de que las variables de tipo I suelen tener abundantes marcas en la matriz variacional, mientras que la situación en la misma zona es más inestable para el tipo $\mathrm{II}^{33}$.

31 “El análisis semántico muestra que el contexto de prótasis de condicionales no llega a neutralizar las distinciones semánticas que señalan el imperfecto de subjuntivo, el condicional y el presente de indicativo. Si bien la elección entre una y otra forma no afecta en general las condiciones de verdad de la oración, es cierto que cada uno de estos tiempos verbales significa en ese contexto un grado distinto en cuanto a la posibilidad de que la acción expresada en la prótasis llegue a tener lugar" (LAVANDERA 1984, p. 33).

${ }^{32}$ Considérense afirmaciones como éstas: "La mayor o menor especificación del referente puede también alterar la selección del modo. Ya es un hecho comprobado que a mayor arbitrariedad del referente, mayor posibilidad de aparición del infinitivo" (MORALES 1989, p. 36); en cuanto a +pg, "los enlaces referenciales que se establecen en cada secuencia... tienen un papel relevante en su caracterización estructural, pero el hecho es que, a su vez, responden, muchas veces, no sólo a la estructura oracional que conforman sino a la secuencia referencial que se ha establecido en todo el párrafo" (1989, pp. 29-30).

${ }^{33}$ Quedaría como problema para esta tipología la variable $\langle 1 d\rangle$, a la que se asignó +st. No niega esto lo anotado en la Tipología 2, pero es obvio que es una dificultad para caracterizar el tipo II sólo como +st. 
Cuadro 3

Variables de tipo funcional

\begin{tabular}{|c|c|c|c|c|c|c|c|c|c|c|c|}
\hline & $<v a r\rangle$ & [vtes] & $f n$ & $m f$ & $s t$ & $s m$ & $p g$ & $h s$ & $g f$ & se & es \\
\hline$\langle a\rangle$ & dequeísmo & que/de que & - & - & + & - & - & + & + & + & ? \\
\hline$\langle b>$ & $\begin{array}{l}\text { sujeto pronominal } \\
\text { presente } \\
\text { expresión del suie- }\end{array}$ & $\mathrm{pr}$ & - & + & ? & - & + & - & - & $?$ & $?$ \\
\hline$\langle c\rangle$ & $\begin{array}{l}\text { expresión del suje- } \\
\text { to }\end{array}$ & pres./aus. & - & + & + & + & + & - & - & - & ? \\
\hline$<d>$ & $\begin{array}{l}\text { personalización de } \\
\text { haber }\end{array}$ & $\begin{array}{l}\text { impers./ } \\
\text { pers. }\end{array}$ & - & + & + & - & - & + & + & + & + \\
\hline$\langle e\rangle$ & $\begin{array}{l}\text { personalización de } \\
\text { hacer }\end{array}$ & $\begin{array}{l}\text { impers./ } \\
\text { pers. }\end{array}$ & - & + & + & - & - & + & + & + & + \\
\hline$<f>$ & $\begin{array}{l}\text { clítico no } \\
\text { argumental }\end{array}$ & pres./aus. & - & - & + & - & + & ? & + & + & + \\
\hline $\begin{array}{l}<g> \\
<h>\end{array}$ & $\begin{array}{l}\text { clítico los } \\
\text { clíticos pleon }\end{array}$ & lo/los & - & + & + & - & - & + & + & $?$ & $?$ \\
\hline & & pre & - & - & + & - & - & $?$ & + & + & $?$ \\
\hline$\langle i\rangle$ & $\begin{array}{l}\text { duplicación de } \\
\text { clíticos }\end{array}$ & pres./aus. & - & - & + & - & + & + & + & - & $?$ \\
\hline$<j>$ & $\begin{array}{l}\text { número de argu- } \\
\text { mentos en verbos } \\
\text { de 'decir' }\end{array}$ & $1 / 2 / 3$ & - & - & + & + & + & - & - & - & $?$ \\
\hline$\langle k\rangle$ & $\begin{array}{l}\text { relación entre dos } \\
\text { proposiciones }\end{array}$ & $\begin{array}{l}\text { yuxt./coor./ } \\
\text { subor. }\end{array}$ & - & - & + & + & + & + & - & + & + \\
\hline$\langle l>$ & voz & \pm activa & - & + & + & $?$ & + & + & - & - & + \\
\hline
\end{tabular}

Son también muy variados los problemas que plantea la lectura del tercer cuadro. De modo semejante a como ocurría con los cuadros 1 y 2, la marca -fn es general. En parcial diferencia con el cuadro 2 y a semejanza del 1, casi todas las variables son -sm; también, como en el cuadro 1 y no en el 2 , se observa una proporción mayor de marcas " + " en la matriz variacional. Existe, por tanto, cierto "eco" tipológico entre los cuadros 1 y 3 .

Una distribución bastante clara es la que presentan las variables $\langle d\rangle$ y $\langle e\rangle$, en contextos como los de (45). Se han considerado variables de clase funcional porque en los dos casos se reinterpreta el CD como sujeto (aunque existen ciertas semejanzas con la variable $\langle 2 j\rangle$, lo que encuentra reflejo en las respectivas matrices gramaticales; las diferencias se localizan en la matriz variacional):

(45) a. Aquí han habido muchas asociaciones de vecinos 
b. Llevamos relaciones desde hacen seis años

(Navarro Correa 1992, pp. 97 y 98)

La reestructuración de argumentos explica la marca +st, y la forma plural del verbo la + mf. Podrían discutirse las asignaciones negativas en las casillas de cambio de significado argumentando, por ejemplo, que existe tendencia a asignar la función sujeto a los referentes animados, como podría defenderse en (45a). Sin embargo, si la animación es una jerarquía, en ambos ejemplos el único argumento es el interpretado como sujeto, y no cabe entonces hablar de jerarquía; en segundo lugar, y es más importante, en (45b) el referente del argumento reinterpretado no es animado. Por último, dada la conocida y fuerte vinculación del objeto al verbo, estas interpretaciones suponen pasar por encima de relaciones claramente establecidas. No vemos, hasta ahora, razón para no asignar -sm y -pg. Resulta notable en estas dos variables la generosidad de la matriz variacional, cubierta por completo.

Tres variables que presentan una estructura muy próxima a estas dos primeras son la $\langle a\rangle$, dequeísmo, la $\langle g\rangle$, función del clítico los, y la $\langle h\rangle$, elítieos pleonásticos.

La variable $\langle a\rangle$ da cuenta de este tipo de ejemplos:

(46) a. Me enteré $\emptyset$ que María llamó

b. Yo digo de que esto es cierto

(Bentivoglio 1980-1981, pp. 704 y 707)

Se ha tomado esta variable como funcional pensando en que $d e$ podría interpretarse como una marca de relación más explícita que $\emptyset^{34}$. Se ha dicho, por otra parte, que las construcciones con

34 Son trabajos pertinentes para la recta comprensión de estos datos Rabanales 1974, Bentivoglio 1976 y 1980-1981 y Bentivoglio y D'INTRONo 1977. Diversos hechos favorecen la interpretación +sc: " $a$ ) las mujeres son más dequeístas que los hombres; $b$ ) los hablantes entre 36 y 55 años de edad... son más dequeístas" (BENTIVOGLIo 1980-1981, p. 709, comentando el trabajo de 1976). Debe considerarse, sin embargo, que algunos comentarios están fundamentados en un número pequeño de datos (Bentivoglio, por ejemplo, está manejando sólo 15 casos de dequísmo en 12 hablantes, de 50). En cuanto a +gf, sí parece haber diferencias geográficas justificatorias: Chile sería más dequeísta que Venezuela. En la recolección de BENTIVOGLIO y D'INTRONo de 1977 , socioeconómicamente estratificada, se encontró más dequeísmo en los hablantes medios, la menor frecuencia era la de los hablantes del nivel bajo, y quedaban entre medias los hablantes del 
$d e$ tienen un significado diferente (distanciamiento, poco compromiso) (García 1986), pero esa misma opinión ha sido sometida a crítica (López Morales 1989, pp. 96-99). Por todo ello nos ha parecido oportuno asignar $-\mathrm{sm},-\mathrm{pg}$.

Un fenómeno muy frecuente en México afecta a la atracción experimentada por el clítico los en ejemplos como el siguiente:

(47) Ustedes tenían que haber traído los libros y los cuadernos. Ya se los advertí

Es decir, podríamos imaginar un par de variantes de este tipo:

(48) a. Les dije que vinieran pronto. Ya se lo dije

b. Les dije que vinieran pronto. Ya se los dije

No parece que haya problemas en marcar $-\mathrm{sm}$ y $-\mathrm{pg}^{35}$. Al igual que varios otros de los casos de variables funcionales que estamos revisando, $\langle g\rangle$ presenta una matriz variacional rica.

La variable <clíticos pleonásticos > alude a los casos estudia-

nivel alto; los hombres son más dequeístas que las mujeres (a diferencia del otro análisis mencionado); de que sería en general más prestigioso; "la frecuencia con que un determinado verbo ocurre y las probabilidades de una construcción con $\mathrm{DQ}$ son inversamente proporcionales: menor es la frecuencia y más alto el porcentaje de DQ, y viceversa"' (BENTIVOGLIo 1980-1981, p. 716). ?es nos abre la duda de si es posible marcar un estilo más formal por medio del uso de la forma de que. En cuanto a + hs, describe lo que parece ser alguna clase de cambio en marcha, pero véase también BOGARD y COMPANY 1989.

${ }^{35}$ Existe una diferente distribución de correferencialidad, pero tomadas en conjunto ambas cadenas de clíticos, no parece haber diferencias de significado. Lo interesante de esta variable es que parece establecer un curioso sistema de correferencias:

a. Le di a Juan un libro. Ya se lo di.

b. Les di a Juan y a Pepe un libro. Ya se los di.

c. Le di a Juan dos libros. Ya se los di.

d. Les di a Juan y a Pepe dos libros. Ya se los di.

Tenemos ahí las cuatro combinaciones posibles de singular y plural para CD y CI. El caso crítico, como es sabido, es el que aparece en (b); la explicación hs dice que se trata de un fenómeno de atracción de la concordancia. Con todo, parecería que los se está interpretando como CI en la segunda oración de (b) -en parte, esa posible ampliación funcional es la que nos ha movido a incluir esta variable en el cuadro de funciones. Por otra parte, lo cierto es que la correferencialidad no implica cofuncionalidad. El fenómeno es de gran interés. 
dos por Silva-Corvalán en Santiago de Chile (49a es un caso de clítico pleonástico):

(49) a. Se la estoy pasándosela

b. Nos íbamos a juntar nosotros con esos chiquillos

(Silva-Corvalán 1989, p. 101)

Los casos de presencia o ausencia de clíticos pleonásticos parecen referencialmente sinónimos y tampoco parece haber diferencias discursivas ${ }^{36}$.

Si cinco de las variables del catálogo "funcional" son - sm y $-\mathrm{pg}$, otras 3 han sido marcadas como $-\mathrm{sm}$, +pg. Sea $\langle i\rangle$, la duplicación de clíticos:

(50) a. A mí se me abrió el mundo cuando $l_{\mathrm{i}}$ conocí a Eugenio $o_{\mathrm{i}}$

b. Me tomó la presión (Silva-Corvalán 1989, p. 109)

En (50a) está "presente" la variante de duplicación de clíticos; está "ausente" en (50b). La función de este clítico sería la de marcar la concordancia entre verbo y objeto (cf. Bogard 1992), "motivada por el relativo valor de topicalidad de la frase nominal acusativa o dativa'" (Silva-Corvalán 1989, p. 115) ${ }^{37}$.

Un ejemplo mexicano que nos ha llamado la atención es éste:

(51) a. Mil pesos le cuesta la agenda billetera de lujo

b. La agenda le contiene calendario

Por oposición a oraciones semejantes sin el clítico, podría formularse una variable de nombre <clítico no argumental>, por parecernos que ni costar ni contener necesitan de CI. Ese valor no argumental explicaría -sm. Estilísticamente, parece recurso

${ }^{36}$ Silva-Corvalán lo describe como un caso de variación sociosintáctica. "La presencia versus la ausencia de los CPs no afecta al significado referencial de los enunciados... ni parece tampoco conllevar significado pragmático ni estar relacionada con diferencias en la perspectiva funcional de las oraciones" (1989, p. 105).

${ }^{37}$ Ciertamente, es delicado asignar -sm en casos como éste, puesto que esta " concordancia sintáctica entre el verbo y el complemento directo. . . responde a factores semánticos y sintácticos que se relacionan con la noción de topicalidad" (SILva-Corvalán 1989, p. 111). Por otra parte, no parece haber diferencia en cuanto a valores de verdad entre $A$ mi se me abrio el mundo cuando lo conocí a Eugenio y $A$ mi se me abrió el mundo cuando conoci a Eugenio; si ello es así, podría defenderse -sm. 
para introducir al auditorio en primer plano, como si le correspondiera papel de argumento, para marcar su importancia y captar su implicación. Que un elemento funcione "como si" fuera otra cosa parece excelente recurso para alumbrar variables; con probabilidad, ese "como si" está en la base de más de un cambio lingüístico. Quizá pueda ensayarse una nueva generalización referida a los no argumentos:

\section{Generalización 7}

Si la variable afecta a no argumentos, la asignación tenderá a ser - sm

Variables con asignación $+\mathrm{sm}$, +pg son la $\langle b\rangle$, sujeto pronominal presente, la $\langle c\rangle$, expresión del sujeto, y la $\langle j\rangle$, número de argumentos en verbos de 'decir'. $\langle b\rangle$ se refiere a ejemplos de este tipo:

(53) a. ...y entonces yo decidí que sí...

b. ...y $\emptyset$ quiero seguir estudiando... (Bentivoglio 1987)

En cuanto al uso de los sujetos pronominales de primera persona en Caracas,

el análisis multivariado de los datos demuestra que, en el español actual hablado en Venezuela, diferentes referentes, número singular, terminaciones verbales ambiguas, verbos de percepción, cognición, dicción, etc., ningún cambio en el turno de la conversación, y sexo masculino favorecen la variante pronominal de un sujeto de primera persona (Bentivoglio 1987 , p. 63$)^{38}$

$\langle c\rangle$ explica ejemplos como éste:

(54) a. Pepe vio a Luis

b. Estuve con Pepe el sábado. $\emptyset \mathrm{Me}$ dijo que vio a Luis

${ }^{38}$ Se trata de demostrar en ese trabajo que "los factores lingüísticos pesan más que los sociales" (Bentivoglio 1987, p. 13), de lo que es testigo el cuadro de matrices de esta variable. Además, ?es va por no haberse considerado la variable estilo. En cuanto a ?sc, podría haber sido sin complicaciones -se, pues "la diferencia entre hombres y mujeres respecto al uso del pronombre de primera persona es mínima" (p. 57), y la muestra manejada es realmente reducida: 12 hablantes, y "el nivel socioeconómico de los hablantes no influye en el uso de los sujetos pronominales" (p. 58). 
Al igual que en los casos estudiados por Bentivoglio, parece que la expresión del sujeto está reguladá por factores semánticos y pragmáticos ${ }^{39}$. Es más, estos hechos podrían ser generales del español. Esto puede sugerir la siguiente generalización:

\section{Generalización 8}

Si la asignación es $+\mathrm{sm}$, +pg, es más probable que la matriz variacional esté desierta (=marcada " -") en mayor grado

La tercera variable $+\mathrm{sm}$, +pg es la $\langle j\rangle$, el número de argumentos en verbos de decir:

(56) a. Juan le dijo que vendría (3)

b. Juan dijo que vendría (2)

c. Dijo que vendría (1)

Este tipo de ejemplos, estudiados en Martín Butragueño (1993), han de explicarse como $+\mathrm{sm}$, como se deduce de la asignación + sm a las dos variables que se acaba de comentar: si +sm vale para la expresión o no del sujeto -como se estudia en $\langle b\rangle$ y $\langle c\rangle-$, la marca ha de valer también para el caso específico de los verbos de 'decir', y lo mismo podría apuntarse de los otros argumentos del verbo ${ }^{40}$; la asignación +pg también es muy clara: por ejemplo, el argumento $\mathrm{CD}$ es el más abundante, puede decirse que acompaña siempre a V: él es, casi siempre, el responsable de introducir la información nueva.

Finalmente, un par de variables que quedan un poco al margen en el cuadro son la < relación entre dos proposiciones $>$ y la $<$ voz $\rangle$. Parece obvio que dadas

(57) a. Juan es amigo de Luis

b. Juan es amigo de Pepe

y

c. Juan es amigo de Luis y es amigo de Pepe

d. Juan es amigo de Luis porque es amigo de Pepe,

39 Véase Silva-Corvalán 1989, pp. 115-117: "la posición y la expresión del sujeto tienen correlación con factores de tipo semántico y pragmático" (p. 115).

${ }^{40}$ Influyen otros factores, entre ellos el propio verbo. 
el significado de (57c) y (57d) es muy diferente; de ahí la asignación $+\mathrm{sm}^{41}$.

En cuanto a la $<\mathrm{voz}>$, que podemos leer en variantes como +activa y -activa, debe recordarse la polémica suscitada a raíz del artículo de Weiner y Labovi2 ${ }^{42}$ En todo caso, si disponemos de ejemplos como

(58) a. Un coche atropelló a Juan

b. Juan fue atropellado por un coche,

41 "La yuxtaposición y la coordinación, como formas más simples de la sintaxis oracional, son bastante más comunes en el habla popular que en la culta. La subordinación se emplea, consecuentemente, más en el habla de las personas de mayor instrucción. Las frecuencias son muy reveladoras: el habla culta se sirve de la subordinación en un $48.2 \%$ de los casos, en tanto que el habla popular sólo lo hace en el $30.3 \%$ de ellos. Esto significa que la norma culta utiliza la subordinación... casi un $60 \%$ más que el habla popular..." (LOPE BLANCH 1987, p. 77).

${ }^{42}$ Ante el problema de si la activa y la pasiva significan lo mismo, WeINER y LABOV comentan: "One strategy... is to say that we are using 'rough semantic equivalence'... But it seems to us that this concession to an idealistic semantics is needlessly unrealistic. If we isolate words from their use, we can show that there is no such thing as a precise synonym, since all words have slightly different privileges of ocurrence when we consider every possible context. But in practice, the need for stylistic variation leads all speakers and writers of English to substitute one word for the other with the expectation that any differences that might arise in other contexts will not affect interpretation in that one" (1983, p. 30). Es interesante considerar que "cuando se producen situaciones que contradicen la LPA [Ley de la Preeminencia de la Animación] solemos obtener estructuras diatéticamente marcadas: pasivas, reflexivas, medias, impersonales, antipasivas y otras muchas" (MOREno Cabreka 1987, p. 48); en cuanto al análisis de la pasiva, "puede caracterizarse como un procedimiento sintáctico que permite colocar como suieto de un verbo agentivo el argumento que desempeña el papel temático de TEMA o PACIENTE"' (BRUCART 1990, p. 195). Debe insistirse en que aquí no nos interesa tanto el análisis de la construcción como el examen de la posibilidad de considerar en un par de activa y pasiva, al menos en algunos casos, variantes de una misma variable. Por otra parte, se ha observado que "originariamente, la noción de variahle... se introdujo para dar cuenta de aquellos casos de variación para los que puede demostrarse que tienen significación social y estilística, que se manifiesta por diferencias consistentes de frecuencias que covarían con otros factores lingǘsticos y extralingüísticos. La única de estas características que se preserva en un análisis como el de Labov y Weiner es la de que las frecuencias covarían con otros elementos, limitados en este caso a formas de la estructura lingüística de superficie"' (LAVANDERA 1984 , p. 44$)$. 
debe reconocerse que intervienen factores morfológicos (en el verbo), sintácticos, quizá pueda discutirse algo respecto de los semánticos, y la lectura temática es, en principio, diferente ( + pg). Es más, para explicar lo que de variables tienen estos dos fenómenos (sin negar otras explicaciones para otros intereses), preferiríamos acudir a la Generalización 4, que puede leer los hechos en términos discursivos y estilísticos.

Así las cosas, y dejando un poco al margen a las variables $\langle k\rangle$ y $\langle l\rangle$, el cuadro de variables funcionales podría tener este (vago) perfil general:

Tipologia 3 (provisional)

Tipo III: +st, \pm sm, \pm pg, desglosable, en realidad, en tres subtipos de contornos más claros:

Tipo Illa: $-\mathrm{sm},-\mathrm{pg}$

Tipo Illb: $-\mathrm{sm}$, + pg

Tipo IIIc: + sm, +pg

En cuanto a las condiciones, todas las variables del tercer cuadro cumplirían la Condición 1; esto especialmente si entendemos el estilo en el sentido (38b) ya comentado, porque si lo entendemos como (38a), muy probablemente $\langle b\rangle,\langle c\rangle$ y $\langle k\rangle$ (obsérvese que todas son $+\mathrm{sm},+\mathrm{pg}$ ) tuvieran dificultades para sobrellevar esta condición, y sólo aceptarían la 3, más general. En cuanto a la Condición 2, el subtipo Illa la aceptaría estrictamente, el Illb sólo de modo relajado, y el IIIc, simplemente, no la aceptaría. Por fin, en principio, todas han sido construidas aceptando la Condición $4^{43}$.

${ }^{43}$ Entre otras, hubiera podido incluirse en este apartado la variable observable en ejemplos como

a. Los papeles los reciclan (desde hace unos años)

b. Los papeles se reciclan (desde hace unos años) (MORALEs 1992, p. 673)

"Las estructuras A y B presentan ciertas diferencias, diferencias relacionadas con la opacidad del agente, la B (estructura con se) parece alejarse un tanto más de éste. La marca de persona en el verbo en la estructura A permite interpretar con un grado mayor de intensidad la participación de dos argumentos (sujeto y objeto), esto comunica a la construcción un grado mayor de transitividad. Esas diferencias son las que ocasionan las distintas variedades dialectales, cierta particular modalidad del discurso, que viene dada por la preferencia de una u otra forma en los contextos restringidos"' (MORALES 1992, p. 684). Otra variable muy interesante es el uso anafórico singular de le en algunas zonas:

a. Le dije a los muchachos que vinieran 


\section{Cuadro 4}

Variables de tipo posicional

\begin{tabular}{|c|c|c|c|c|c|c|c|c|c|c|c|}
\hline & $<v a r>$ & [vtes $]$ & $f n$ & $m f$ & $s t$ & $s m$ & $p g$ & hs & $g f$ & se & es \\
\hline$\langle a\rangle$ & orden verbo-sujeto & SV/VS & + & - & + & - & + & - & - & - & $?$ \\
\hline$\langle b\rangle$ & $\begin{array}{l}\text { orden verbo- } \\
\text { complemento }\end{array}$ & $\mathrm{GV} / \mathrm{VC}$ & + & - & + & - & + & - & - & - & ? \\
\hline$\langle c\rangle$ & posición del clítico & $\begin{array}{l}\text { Clít.-V/ } \\
\text { V-Clít. }\end{array}$ & + & - & + & - & + & - & - & - & $?$ \\
\hline$<d>$ & $\begin{array}{l}\text { complemento } \\
\text { preverbal }\end{array}$ & $\begin{array}{l}\text { enlace/foco } \\
\text { contr./con- } \\
\text { trario/comp. } \\
\text { focal }\end{array}$ & + & - & + & - & + & - & - & - & $?$ \\
\hline$\langle e\rangle$ & $\begin{array}{l}\text { tipo de perífrasis } \\
\text { de relativo }\end{array}$ & tipo $\mathrm{a} / \mathrm{b} / \mathrm{c}$ & + & - & + & - & + & $?$ & - & - & $?$ \\
\hline$<f>$ & $\begin{array}{l}\text { orden verbo- } \\
\text { complemento } \\
\text { directo en verbos } \\
\text { de 'decir' }\end{array}$ & $\begin{array}{l}\text { CD-V/ } \\
\text { V-CD }\end{array}$ & $(+)$ & - & + & - & + & - & - & - & $?$ \\
\hline$<g>$ & $\begin{array}{l}\text { orden verbo-suje- } \\
\text { to-complemento } \\
\text { directo en verbos } \\
\text { de 'decir' }\end{array}$ & $\begin{array}{l}\text { S-CD-V/ } \\
\text { S-V-CD/ } \\
\text { CD-S-V/ } \\
\text { CD-V-S/ } \\
\text { V-S-CD/ } \\
\text { V-CD-S }\end{array}$ & $(+)$ & - & + & - & + & - & - & - & $?$ \\
\hline$\langle h\rangle$ & orden Adj-N & $\begin{array}{l}\text { Adj-N/ } \\
\text { N-Adj }\end{array}$ & + & - & & $(-)$ & + & - & - & - & + \\
\hline$\langle i\rangle$ & $\begin{array}{l}\text { orden de los modi- } \\
\text { ficadores ante } \mathrm{N}\end{array}$ & $\begin{array}{l}\text { la su } \mathrm{N} / l a \\
\text { esta, su } \mathrm{N} / l a \\
\mathrm{~N} \text { suya }\end{array}$ & $(+)$ & - & + & $?$ & $?$ & + & $(+)$ & $(+)$ & $(+)$ \\
\hline
\end{tabular}

El cuadro 4, afortunadamente, parece tener una lectura mucho más clara, por lo menos en líneas generales. La asignación típica en este cuadro parece ser

$$
<\text { var }>\rightarrow\{[\mathrm{a}],[\mathrm{b}] \ldots\},\left[\begin{array}{ll}
+\mathrm{fn} & -\mathrm{mf} \\
+\mathrm{st} & -\mathrm{sm} \\
+\mathrm{pg} &
\end{array}\right]\left[\begin{array}{ll}
-\mathrm{hs} & -\mathrm{gf} \\
-\mathrm{sc} & +\mathrm{es}
\end{array}\right]
$$

En prácticamente todas las variables de este cuadro la asignación de la cima melódica parece ser de interés $(+\mathrm{fn})$, y hecho tan constante merecería intentar una generalización como ésta:

b. Les dije a los muchachos que vinieran (agradecemos la observación al prof: Max E. Figueroa). 


\author{
Generalización 9 \\ $\mathrm{V}<\mathrm{var}>$ gramatical, $\alpha \mathrm{pg}, \alpha \mathrm{fn}$
}

Lo que debe leerse como la tendencia que $|\mathrm{fn}|$ y $|\mathrm{pg}|$ tengan el mismo signo. Esto, que no es demasiado problemático en este cuadro, puede volverse más enrevesado al revisar otros, desde luego; sin embargo, pudiera inducir a revisar algunas asignaciones de signo y, en cualquier modo, a intentar explicar los casos que no se ajusten a la generalización.

En todo caso, tan repetido es el esquema, que podemos proponer como "tipo" provisional uno que fuera:

$$
\begin{aligned}
& \text { Tipologia } 4 \text { (provisional) } \\
& \text { Tipo IV: +fn, +st, +pg, }
\end{aligned}
$$

dando, como siempre, prioridad a las marcas positivas. Salta también a la vista la precariedad de la matriz variacional, casi siempre desierta. Una vez más, |es| se interpretará casi siempre en el sentido de (38b), lo que no va contra la Generalización 6; es más, existe siempre la tentación de interpretar algunas variables según la Generalización 4 (referida a las repercusiones discursivas de las variables establecidas sobre categorías mayores). Tendríamos, entonces, un conjunto de variables que a duras penas cumplen la Condición 1, salvo en el sentido de |es| como (38b), aunque sí cumplen la Condición 3 (y, en principio, también la 4). A la hora de revisar las variables, el problema no es tanto justificar + pg, que suele ser bastante claro, como probar la licitud de asignar $-\mathrm{sm}$, siquiera provisionalmente ${ }^{44}$.

44 Aunque la conclusión a que suele llegarse en trabajos que se ocupan de variables posicionales es que los factores reguladores de la distribución de las variantes son fundamentalmente de naturaleza semántico-pragmática, lo cierto es que se suele partir de preguntas de carácter sintáctico. La pregunta de partida es de este tipo: “¿qué factores regulan la posición de tales funciones sintácticas?"; los problemas estudiados son, por ejemplo, “¿qué factores regulan la distribución de las variantes $[\mathrm{S}-\mathrm{V}],[\mathrm{V}-\mathrm{S}]$ ?", " “qué factores regulan la distribución de las variantes [CI-V], [V-CI]?”, entre muchas otras. En ellas lo fundamental es el orden de funciones sintácticas tipo \{sujeto, complemento directo... \}. De hecho, se analizan las posibles funciones pragmáticas de tal función sintáctica en tal posición. Los hechos se han planteado a veces de modo algo diferente. Ya en Contreras 1983 (1976) se plantea que funciones informativas como \{tema, rema, tópico\} se asignan no sobre la estructura sintáctica, sino sobre la estructura semántica formada por funciones como \{agente, paciente...\}: “La jerarquía remática está determinada por 
Respecto a la variable $\langle a\rangle$, referida al orden entre verbo y sujeto, Ocampo (1990) afirma que son tres los tipos de factores que afectan al orden de palabras: "(a) information factors (New/Old information), (b) pragmatic functions (contrary to expectation, focus of contrast, etc.), and (c) verb type (onevalency, two valency)" (p. 87)45. Para Delbecque (1992) la "anteposición y posposición del regente [SN que rige la persona y número del verbo] se oponen básicamente por manifestar esque-

la estructura semántica de la oración. Esto explica que dos verbos intransitivos como empezar y fracasar impongan una distinta selección de rema a sus oraciones, y que dos verbos con diferentes propiedades sintácticas, pero semejantes en cuanto a sus propiedades semánticas como crecer y parecer den prioridad al mismo elemento semántico, la cláusula paciente" (1983, p. 50). Además, según Contreras, el orden de palabras y la colocación de la cima melódica pueden estudiarse sin necesidad de contar con factores extralingüísticos (p. 22) - piénsese en la precariedad de la matriz variacional en el cuadro 4; es más, muchas de las supuestas alternancias estilísticas no significan realmente lo mismo (p. 43). De ser ciertas las afirmaciones de Contreras, tendríamos una variable más semántica que sintáctica (sólo subsidiariamente sintáctica). Quizá elimináramos muchos de los problemas estudiando, por ejemplo, la posición de los agentes respecto a los pacientes. Esto, por otra parte, ayudaría a asegurar la equivalencia semántica de las variantes. La pregunta pertinente sería: “¿qué factores regulan la posición de tales funciones semánticas?", que puede ejemplificarse así: “¿cuál es la asociación en un texto entre [ + agente] y [ + rema]?"' Puede que este planteamiento adquiera su pleno sentido si se piensa, por ejemplo, en jerarquías como la de topicalidad propuesta por Givón 1976. Un par de estudios sobre el español que la toman en cuenta son Silva-CoRVALÁN 1989, pp. 109-115 y BOGARd 1992; muy clarificador es Givón 1988.

${ }^{45}$ Habría una correlación entre función pragmática, orden de palabras y posición del acento primario. Por otra parte, conviene señalar que muchos de los factores mencionados por OCAMPO 1990 aparecen una y otra vez en los trabajos que se ocupan de variables posicionales. Cuando se considera el factor información, la autoridad suele ser el ya clásico trabajo de PRINCE 1981, habitualmente bastante simplificado en sus conceptos para poder trabajar con datos reales, procedentes de algún corpas. Además, es importante considerar que "information factors and pragmatic functions are two independent principies, not reducible to the same category. The difference between them is that the first ones refer to the status of a NP referent in the mind of the hearer, whereas the second ones designate a relationship between a referent and a proposition, or between a proposition and its context" (OcAMPo 1990, p. 91). En el trabajo que estamos comentando no se consideraron, al parecer, las categorías incluidas en la matriz variacional; nos sentimos tentados a interpretar que el autor consideró que ese tipo de factores no eran importantes para el caso. 
mas cognitivos distintos" (p. 63) ${ }^{46}$. Parecidos factores son los que contribuyen a explicar la distribución de la mayoría de las variables posicionales. Así, otro tanto puede decirse de la variable $\langle b\rangle$ :

(63) a. Al vecino le robaron el auto (CI preverbal)

b. A mi hermano lo llevaron al circo (CD preverbal)

c. Le robaron el auto al vecino (CI posverbal)

d. Llevaron a mi hermano al circo (CD posverbal) (SilvaCorvalán 1989, pp. 117, 118)

El análisis de Silva-Corvalán parece mostrar en la matriz gramatical una distribución semejante al de Ocampo para el orden verbo-sujeto; y en cuanto a la matriz variacional, no parecen existir correlaciones sociales claras, lo que deja vana esta sección de casillas. Vamos a ver repetido el esquema en variables un poco más específicas, como la $\langle f\rangle$, referida al orden de $\mathrm{V}$ y complemento en verbos de 'decir'47; por cierto que la marca $(+)$ fn se justifica por la filiación escrita del corpus sobre el que se constituyó esta variable; el factor es, presumiblemente, activo en la lengua hablada e incluso en la lectura. Todo esto es también válido para la variable $\langle g\rangle$, que examina el orden verbo-sujeto-CD en verbos de 'decir' (con seis posibles variantes):

(64) a. Los asesores del ex presidente han dicho que esperan que la próxima semana se discuta la posibilidad de que Gorbachov declare en privado (sujeto-V-CD)

b. "Es una barrera no arancelaria", dijo Pablo Cuarón (CD-Vsujeto) (Martín Butragueño 1993, p. 7)

46 De hecho, "se ha verificado la tendencia a empezar el enunciado con los elementos + tópico y ya presentes. Pero el hecho de que a veces no se cumpla, y que un regente + tópico, cuyo referente ya está presente, pueda posponerse, indica que la organización textual queda supeditada al condicionamiento de la gramática discursiva: para obtener una lectura -agentiva se prefiere la posposición. Al revés, la anteposición de un regente -tópico y/o ausente del contexto anterior, se explica por el deseo de acercarlo en la medida de lo posible al polo + agentivo" (Delbeceue 1992, p. 45, n. 56). Dada la asociación entre posición, agentividad, topicalidad e introducción del referente, probablemente sería necesario asignar $+\mathrm{sm}$, + pg.

${ }^{47} \mathrm{El}$ orden predominante fue el VO, pues con independencia de la presencia o no de otros argumentos, se documentó en 1585 ocasiones $(70.44 \%)$, frente al orden OV (612 casos, 27.2\%) (MARTín Butragueño 1993, p. 8). 
Cuando se ha estudiado la distribución de las funciones discursivas de un elemento situado en una posición dada, los factores interesantes parecen también ser los mismos. Así, SilvaCorvalán (1989, pp. 121-128) ha estudiado la multifuncionalidad del objeto preverbal (variable $\langle d\rangle$ ), considerando cuatro variantes (enlace contextual, foco contrastivo, contrario a lo esperado y complemento focal); no parece haber razones para establecer marcas " + " en la matriz variacional, a excepción del estilo en el sentido de (38b); es más, este tipo de hechos van más allá de los límites del español ${ }^{48}$. Podríamos incluso preguntarnos si es correcto asignar esta clase de distribución de marcas a variables como $\langle c\rangle$, referida a la posición del clítico y representante de ejemplos como éste:

(65) a. Fui a verlo b. Lo fui a ver

De ser correcta la interpretación, reforzaría la idea de la semejan$z a$ en la naturaleza de los fenómenos posicionales ${ }^{49}$. Otro ejemplo interesante es el de las perífrasis de relativo (variable $\langle\ell\rangle$ ):

(66) a. Fue a Juan al que elegimos

b. A Juan fue al que elegimos

c. Al que elegimos fue a Juan

(Moreno Cabrera 1983, pp. 455-456) $)^{50}$,

con también, en principio, una matriz variacional despoblada.

Otra variable muy interesante es el orden entre adjetivo y sustantivo $^{51}$. Se ha defendido (Delbecque 1990) la importancia del factor tematicidad para regular la posición del adjetivo español.

48 Silva-Corvalán subraya que "el orden de palabras en español no es libre sino controlado por factores semántico-pragmáticos. Las condiciones bajo las cuales un objeto aparece en posición inicial pueden identificarse si nuestra metodología va más allá del análisis intraoracional y toma en cuenta la relación entre sintaxis, prosodia y significado pragmático" $(1989$, p. 128).

${ }^{49} \mathrm{Cf}$., entre otras referencias, MYHILL 1989.

${ }^{50}$ Para algunos recuentos, véase García Mouton, Martín ButragueÑo y Molina Martos 1991.

${ }^{51}$ Marta Luján defendía en 1980 que "la mayoría de los adjetivos, cualesquiera sea su posición en la estructura superficial, derivan de adjetivos predicativos en la estructura profunda"' (p. 17). Y se decía abiertamente que, fuera delante o detrás del sustantivo, "en la mayoría de los casos, el significado del adjetivo es el mismo" (p. 81). 
Aunque haya cambios de significado para algunos adjetivos según vayan delante o detrás del sustantivo, como en el caso de viejo o grande (de ahí la marca (-)sm), el hecho es que no basta con la semántica de la palabra para explicar ejemplos como éstos:

(67) a. Un problema grave / un grave problema

b. El agente principal / el principal agente

(Delbecque 1990, p. 350)

Un poco al margen en su distribución, pero muy interesante por su carácter histórico (lo que explicaría su "rareza" matricial) queda la variable $\langle i\rangle$, que examina los cambios en el orden de los modificadores antepuestos al sustantivo. Ha habido dos clases de cambios, unos en la frecuencia y otros en la estructura (Company 1991). La marca (+)fn explicaría el cambio de la su casa a la casa suya, si es que han de estar presentes los dos elementos. Ignoramos la importancia exacta de los factores semánticos y pragmáticos ${ }^{52}$. Por otra parte, se ha marcado $(+) g f,(+) s c$ y $(+)$ es imaginando un proceso de difusión del cambio lingüístico. En todo caso, se revela una vez más la interesante distribución de los fenómenos variables históricos, y la necesidad de distinguir en $|h s|$ dos sentidos,

(68) a. El histórico propiamente dicho y

b. El referido a los procesos de cambios en marcha, inducidos a través de diferentes pruebas

Quizá el hecho más característico de las variables posicionales, dejando a un lado la discusión del problema de asignar + o -sm (véase n. 46), sea la escasez de marcas positivas en la matriz variacional. Si adoptáramos un criterio estricto de variable sociolingüística, nos veríamos obligados a confesar el dudoso interés (a posteriori) de estas variables para la sociolingüística. En todo caso, podríamos formular generalizaciones de este tenor:

Generalización 10

De una variable posicional cabe esperar que sea -se

52 Resulta interesante la constatación de que "no son relevantes las diferentes funciones que aquélla [la frase sustantiva] puede realizar en la oración" (COMPANY 1991, p. 55). 
Naturalmente, sería muy interesante encontrar casos de variables posicionales $+\mathrm{sc}$ y explicar por qué se producen.

\section{Conclusiones}

Para concluir, nos gustaría recordar las ventajas de la explicitud. Ése es el primer objetivo de las matrices de variables construidas y de las condiciones, hipótesis y generalizaciones intentadas. Existen, sin duda, muchos puntos discutibles en la asignación de las marcas; esto es problema, en primer lugar, del estado actual de las investigaciones y, en segundo lugar, de nuestra interpretación de la naturaleza y sentido de esos hechos. Además, es necesario continuar aumentando el repertorio de variables, y confrontar sus características con las generalizaciones trazadas. Es muy probable que cierto número de hechos particulares puedan explicarse por principios de tipología universal. Por ejemplo, la posición preferencial del tópico o la del agente están en la base de la explicación de buen número de variables. En realidad, generalizaciones como la 10, que afirman el probable carácter -se de las variables posicionales, son bastante comprensibles, si se considera que muchos de los factores que afectan a la movilidad de orden son comunes a las lenguas en general.

Un problema difícil es el de dar contenido exacto a las diferentes columnas: $\mathrm{fn}, \mathrm{mf}$, etc., y en especial el de discutir unas fronteras coherentes para zonas como st, sm, pg. Decir eso parece significar haber avanzado poco, pero es imprescindible esta permanente vuelta atrás. De hecho, el mejor conocimiento de los factores condicionantes de un fenómeno puede inducir a revisar las marcas de asignación en las matrices; y tal revisión exige, a su vez, el examen de las varias generalizaciones que puedan haberse visto afectadas o no por los nuevos cambios.

Conviene recordar que el punto de vista de la variación lingüística no es el mismo que el de la gramática. Más que tratarse de métodos alternativos, se ocupan de cuestiones diferentes. Si pensamos en las oraciones activas y pasivas, la pregunta como gramáticos puede ser si se debe o conviene relacionarlas explícitamente. Pero como estudiosos de la variación, la pregunta es si al hablante se le ofrecen ambas posibilidades en el mismo plano de elección, y cuáles son las repercusiones de esa elección. Desde luego, todas estas cuestiones pueden estar relacionadas, pero lo 
importante ahora es subrayar la naturaleza diferente de las preguntas. De ahí el modo en que está planteado este trabajo.

La principal dificultad, en nuestra opinión, no es discutir si las variables han de ser $\pm \mathrm{sm}$ o $\pm \mathrm{pg}$, con tal que cada estudio sea coherente con las características de su tipología, sino más bien explicar por qué tales variables son $+\mathrm{sm}$ o $-\mathrm{sm}$.

Se ha venido configurando la siguiente tipología a lo largo del trabajo:

(70) Tipología 5 (tentativa)

a. Tipo I: $+\mathrm{mf}+\mathrm{hs}+\mathrm{gf}+\mathrm{sc}$

b. Tipo II: + st

c. Tipo III: +st, \pm sm, \pm pg, desglosable, en realidad, en tres subtipos de contornos más claros:

Tipo Illa: $-\mathrm{sm}$, - pg

Tipo Illb: $-\mathrm{sm}$, +pg

Tipo IIIc: + sm, +pg

d. Tipo IV: +fn, +st, +pg

Como puede observarse, se trata sólo de una tipología provisional, pues hay más rasgos de los necesarios para oponer cuatro elementos, y hay oposiciones entre tipos que no están bien confiruradas, como es el caso de los tipos II (70b) y III (70c), entre otras. Además, no faltan variables que quedan peligrosamente ambiguas entre unos y otros tipos. Quizá lo más interesante sea descubrir la homogeneidad patente en grupos de variables próximas; parece improbable que sean casuales las semejanzas entre variables que se ocupan de problemas morfológicos, del cambio de modo o del orden de palabras. Naturalmente, dejar patentes los parentescos no resuelve los problemas, pero sí ayuda a construir nuevas variables dentro de un marco apropiado. Precisamente lo que se ha querido subrayar en cada clase tipológica es lo más típico de esa clase. De ahí que en el tipo I se insista en la relación entre la variación morfológica y la propia de los órdenes de la matriz variacional; por eso en el tipo II se marca como característico sólo el aspecto sintáctico, aislado en general de otros órdenes de variación; la calificación del significado, semántico y pragmático, parece ser lo típico del tercer tipo, aunque asociado a los aspectos sintácticos. Por fin, el tipo IV, bastante más homogéneo, aparece sistemática y simultáneamente asociado a lo fónico, lo sintáctico y lo pragmático.

Uno de los aspectos más llamativos de una tipología de 
variables sociolingüísticas es, precisamente, dejar ver, siquiera sea de manera parcial, qué grupos de variables tienen tal o cual clase de matriz más rica. En especial, es interesante examinar qué tienen en común desde el punto de vista de su matriz gramatical las variables dotadas de una matriz variacional rica, y otro tanto las que muestran una rejilla escasamente cubierta.

Nos parece crucial el papel desempeñado por |es| en todo momento. Su interpretación como 'forma en que se está construyendo el significado' es decisiva para estar seguros del interés de estudiar como variables muchos fenómenos; +es se asocia a + sm y a + pg en múltiples ocasiones. Disponer de una teoría del estilo, o de 'qué podemos hacer para decir lo que queremos decir' (en términos de acción y voluntad) vuelve a estar en el inicio y en el final del problema de la construcción de significados lingüísticos.

Pedro Martín Butragueño Consejo Superior de Investigaciones Cientifficas

El Colegio de México

\section{APÉNDICE}

Algunas DEFiniciones:

(5) "Variacionismo" es la teoría o perspectiva que defiende que el argumento cuantitativo tiene alguna importancia en el estudio del lenguaje

(6) Argumento cuantitativo es, al menos,

a. El que se apoya en la asignación de cantidades $x_{1}, x_{2} \ldots x_{n}$ a variantes $\left[y_{1}\right],\left[y_{2}\right] \ldots\left[y_{\mathrm{n}}\right]$ de una variable $\langle y\rangle:\left[y_{\mathrm{i}}\right] \rightarrow x_{\mathrm{i}}$.

b. El que se construye asignando jerarquías entre variantes $\left[y_{1}\right]$, $\left[y_{2}\right] \ldots\left[y_{n}\right]$ de una variable $\langle y\rangle$. Por ejemplo, $\left.\left.\left[y_{3}\right]\right\rangle\left[\nu_{1}\right]\right\rangle\left[y_{2}\right]$.

c. El que se construye asignando valores de presencia [1] o ausencia $[\emptyset]$ a las variantes $\left[y_{1}\right],\left[y_{2}\right] \ldots\left[y_{\mathrm{n}}\right]$ de una variable $\langle y\rangle$. Por ejemplo, $\left[y_{1}\right] \rightarrow[1] ;\left[y_{2}\right] \rightarrow[\emptyset] ;\left[y_{3}\right] \rightarrow[1]$

(9b) Clase de regla propuesta:

$<y>\rightarrow\{|A|,|B| \ldots|N|\},\left\{\left|A^{\prime}\right|,\left|B^{\prime}\right| \ldots\left|N^{\prime}\right|\right\}$

(38) Dos concepciones de estilo:

a. El estilo en cuanto correlación de otra medida

b. El estilo en cuanto elección para construir el significado

(68) Dos sentidos de hs: 
a. El histórico propiamente dicho y

b. El referido a los procesos de cambios en marcha, inducidos a través de diferentes pruebas

\section{CONDICIONES PROPUESTAS:}

(14) Condición 1 sobre las variables sociolingüiśticas

Una variable sociolingüística ha de tener al menos una marca " +" en cada una de las dos matrices, gramatical y variacional: $\forall\langle x\rangle$ sociolingüística, $\mathrm{E}$ al menos un "+" en $\mathrm{M}_{\mathrm{G}}$ y en $\mathrm{M}_{\mathrm{V}}$

(15) Condición 2 sobre las variables sociolingüisticas

a. Estricta: Una variable sociolingüística lo es si tiene $-\mathrm{sm}$ y $-\mathrm{pg}$ en la matriz gramatical

b. Relajada: Una variable sociolingüística lo es si tiene -sm en la matriz gramatical

(17) Condición 3 sobre las variables

$\mathrm{V}<\mathrm{x}>, \mathrm{E}$ al menos un "+" en $\mathrm{M}_{\mathrm{G}}$ o en $\mathrm{M}_{\mathrm{V}}$

(18) Condición 4 sobre las marcas en las reglas de cualquier clase de variable El argumento decisivo para fijar la marca debe ser el cuantitativo, en el sentido de (6)

Algunas HIPÓtesis:

(16) Hipótesis 1

Una variable lo es si tiene, al menos, una marca " + " en una cualquiera de las dos matrices, gramatical o variacional. Es decir: $\mathrm{V}\langle\mathrm{x}\rangle, \mathrm{E}$ al menos un "+" en $\mathrm{M}_{\mathrm{G}}$ o en $\mathrm{M}_{\mathrm{V}}$

\section{(27) Hipótesis 2}

Si un $Y_{n}$ de la matriz variacional está asociado a " + ", esto implica que $Y_{1}$ a $Y_{n-1}$ están asociados a " + ". Esto es, si un $\left|Y_{n}\right| \in M_{V}$ $\rightarrow$ " + ", entonces $\mathrm{Y}_{1}$ a $\mathrm{Y}_{\mathrm{n}-1} \rightarrow$ " + "

\section{TiPOLOGÍA:}

(19) Tipologia 1 (provisional)

Las variables de tipo I se caracterizan por mostrar $+\mathrm{mf}$

(44) Tipologia 2 (provisional)

a. Tipo I: $+\mathrm{mf}+\mathrm{hs}+\mathrm{gf}+\mathrm{sc}$

b. Tipo II: + st

(59)

Tipología 3 (provisional) 
Tipo III: +st, \pm sm, $\pm p g$, desglosable, en realidad, en tres subtipos de contornos más claros:

Tipo Illa: - sm, - pg

Tipo Illb: - sm, +pg

Tipo IIIc: + sm, +pg

(62) Tipología 4 (provisional)

Tipo IV: +fn, +st, +pg

(70) Tipología 5 (tentativa)

a. Tipo I: $+\mathrm{mf}+\mathrm{hs}+\mathrm{gf}+\mathrm{sc}$

b. Tipo II: +st

c. Tipo III: + st, \pm sm, \pm pg, desglosable, en realidad, en tres subtipos de contornos más claros:

Tipo Illa: - sm, - pg

Tipo Illb: $-\mathrm{sm}$, +pg

Tipo IIIc: $+\mathrm{sm}$, +pg

d. Tipo IV: +fn, +st, +pg

\section{Generalizaciones:}

(23) Generalización 1

En las variables del tipo I hay pocas diferencias entre las que afectan a la esfera nominal y las que afectan a la esfera verbal

(24) Generalización 2

Las variables del tipo I tienden a cubrir con " + " todas las secciones de la matriz variacional

(28) Generalización 3

En el tipo $I$, si un $\left|Y_{n}\right| \in M_{V} \rightarrow$ " $+"$, entonces $Y_{1}$ a $Y_{n-1} \rightarrow$ " + "

(36) Generalización 4

Es más probable que una variable tenga repercusiones discursivas cuanto más altas sean las categorías a las que afecta

(35) Generalización 5

La proximidad de dos casos de la misma variable limita las posibilidades contextuales

(39) Generalización 6 (3 revisada)

Si un $\left|Y_{n}\right| \in M_{Y} \rightarrow$ " +", entonces $Y_{1}$ a $Y_{n-1} \rightarrow$ " +", tomando en cuenta que |es| debe interpretarse como correlación de otra medida

Generalización 7 
Si la variable afecta a no argumentos, la asignación tenderá a ser -sm

Generalización 8

Si la asignación es $+\mathrm{sm}$, +pg, es más probable que la matriz variacional esté desierta (= marcada " - ") en mayor grado

(61) Generalización 9

$\mathrm{V}<\mathrm{var}>$ gramatical, $\alpha \mathrm{pg}, \alpha \mathrm{fn}$

(69) Generalización 10

De una variable posicional cabe esperar que sea - se

\section{BIBLIOGRAFÍA}

Bentivoglio, P. 1976. "Queísmo y dequeísmo en el habla culta de Caracas", en 1975 Colloquium on Hispanic Linguistics. Comps. F. M. Aid et al. Georgetown University Press, Washington, pp. 1-18.

— 1980-1981. "El dequeísmo en Venezuela: ¿un caso de ultracorrección?", BdFS, 31, 705-719.

— 1987. Los sujetos pronominales de primera persona en el habla de Caracas. Universidad Central de Venezuela, Caracas.

— y F. D'Introno 1977. "Análisis sociolingüístico del dequeísmo en el habla de Caracas", BAPuL, 6, 58-82.

BERSCHIN, H. 1987. "Futuro analítico y futuro sintético en el español peninsular y colombiano", $L E A, 9,1,101-110$.

BogARD, S. 1992. "El estatus del clítico de complemento indirecto en español", en Reflexiones lingüisticas y literarias. T. 1: Lingüistica. Eds. R. Barriga Villanueva y J. García Fajardo. El Colegio de México, México, pp. 171186.

- y C. Company 1989. "Estructura y evolución de las oraciones completivas de sustantivo en el español", $R P h, 43,258-273$.

BRUCART, JosÉ MA. 1987. "El modelo de rección y ligamiento", en La elisión sintáctica en español. Universidad Autónoma, Barcelona.

— 1990. "Pasividad y atribución en español: un análisis generativo", en Estudios de lingüistica de España y México. Eds. V. Demonte y B. Garza Cuarón. UNAM-E1 Colegio de México, México, pp. 179-208.

Cheshire, J. 1987. "Syntactic variation, the linguistic variable, and sociolinguistic theory", Ling, 25, 257-282.

Company, Concepción 1991. La frase sustantiva en el español medieval. Cuatro cambios sintácticos. UNAM, México.

COMrIe, BERNARD 1989 [1981]. Universales de lenguaje y tipología lingüistica. Trad. A. Ayuso. Gredos, Madrid.

Contreras, Heles 1983 [1976]. El orden de palabras en español. $2^{\text {a }}$ ed. Cátedra, Madrid.

— 1992. "Principios y parámetros sintácticos", en Reflexiones lingüísticas y literarias. T. 1: Lingüistica. Pp. 139-154. 
DelbecQue, N. 1990. "Word order as a reflection of alternate conceptual construals in French and Spanish. Similarities in adjective position", Cognitive Linguistics, 1, 349-416.

- 1991. Gramática española: Enseñanza e investigación. II: Gramática. 4. El orden de los sintagmas. La posición del regente. Estudio de la variación sintáctica en una perspectiva cognitiva y probabilista. Ediciones Universidad de Salamanca, Salamanca.

— 1992. "Por qué y cómo integrar la variación en la descripción gramatical", LEA, 14, 5-68.

D'INTRONo, E. 1987. “Teoría lingüística, variación paramétrica y español de América", en Actas del I Congreso Internacional sobre el español de América. Eds. H. López Morales y M. Vaquero. Academia Puertorriqueña de la Lengua Española, San Juan, Puerto Rico, pp. 373-382.

Escandell Vidal, M. Victoria 1993. Introducción a la pragmática. AnthroposUniversidad Nacional de Educación a Distancia, Barcelona-Madrid.

García, É. 1986. "El fenómeno (de)queísmo desde una perspectiva dinámica del uso comunicativo de la lengua", en Actas del II Congreso Internacional sobre el español de América. Ed. J. Moreno de Alba. UNAM, México, pp. 46-65.

García FAJARDo, J. 1989. "El sentido en la función referencial de frases sustantivas del español", $N R F H, 37,19-26$.

García Mouton, P., P. Martín Butragueño, e I. Molina Martos 1991. "Sobre el estudio de las construcciones de relativo en el español de América", en El español de América. Actas del III Congreso Internacional de "El espanol de América». Eds. C. Hernández et al. Junta de Castilla y León, Valladolid, pp. 719-727.

Givón, T. 1976. "Topic, pronoun and grammatical agreement", en Subject and topic. Ed. C. Li. Academic Press, New York, pp. 149-188.

— 1988. "The pragmatics of word-order: Predictability, importance and attention", en Studies in syntactic typology. Eds. M. Hammond, E. A. Moravcsik, \& J. R. Wirth. John Benjamins, Amsterdam-Philadelphia, pp. 243-284.

Hernanz, Ma. Lluísa, y José Ma. Brucart 1987. La sintaxis. 1: Principios teóricos. La oración simple. Crítica, Barcelona.

JACOBSON, S. 1989. "Some approaches to syntactic variation", en Language change and variation. Eds. R. W. Fasold \& D. Schiffrin. John Benjamins, Amsterdam-Philadelphia, pp. 381-394.

KLEIN, F. 1979. "Factores sociales en algunas diferencias lingüísticas en Castilla la Vieja", Papers: Revista de Sociología, 11, 45-64.

Labov, William 1972. Sociolinguistic patterns. University of Pennsylvania Press, Philadephia.

— 1978. "Where does the linguistic variable stop? A response to Beatriz Lavandera", Working Papers in Sociolinguistics. Sociolinguistic Working Paper 44. Southwest Educational Development Laboratory, Austin, TX.

LANGACKER, RONALD W. 1987. Foundations of cognitive grammar. T. 1: Theoretical prerequisites. Stanford University Press, Stanford, CA.

LAVANDERA, B. R. 1978. "Where does the sociolinguistic variable stop?", LangS, 7, 171-182.

- 1984. Variación y significado. Hachette, Buenos Aires. 
— 1990. "El cambio de modo como estrategia de discurso", en Indicativo $y$ subjuntivo. Ed. I. Bosque. Taurus, Madrid, pp. 330-357.

Leonetti Jungl, Manuel 1990. El artículo y la referencia. Taurus, Madrid.

Levinson, Stephen C. 1983. Pragmatics. Cambridge University Press, Cambridge.

Lope Blanch, JuAn M. 1987. 'Análisis gramatical del discurso. UNAM, México.

LÓPEZ MORAles, Humberto 1989. Sociolingüistica. Gredos, Madrid.

Luján, Marta 1980. Sintaxis y semántica del adjetivo. Cátedra, Madrid.

Martín Butragueño, Pedro 1992. Desarrollos sociolingüisticos en una comunidad de habla. Tesis doctoral. Reproducción facsímil. Universidad Complutense, Madrid.

— 1993. "Variación sintáctica y semántica en los verbos de 'decir'. Datos del español de México", comunicación presentada al XI Congreso Internacional de la ALFAL, celebrado del 11 al 16 de abril de 1993 en Veracruz, México.

- en prensa. "Él control individual de las reglas sociolingüísticas", $R E L$.

MiLroY, L. 1987. Observing and analysing natural language. Basil Blackwell, Oxford-New York.

MORAles, A. 1989. "Algunas consideraciones sobre la alternancia subjuntivo-infinitivo en las construcciones con para", $N R F H, 37,27-42$. 1992. "Acerca de la topicalidad de objeto en algunos dialectos del español", $R F E, 72,671-685$.

Moreno, F. et al. 1988. "Anotaciones sobre el leísmo, el laísmo y el loísmo en la provincia de Madrid", Epos, 4, 101-122.

- (ed.) 1992. Sociolinguistics and stylistic variation. University of MinnesotaUniversitat de València, Minneapolis, MN-Valencia.

Moreno Cabrera, J. C. 1983. "Las perífrasis de relativo", en Serta Philologica F. Lázaro Carreter. T. 1: Estudios de lingüistica y lengua literaria. Cátedra, Madrid, pp. 455-467.

— 1987. Fundamentos de sintaxis general. Síntesis, Madrid.

MyHILl, J. 1989. "Variation in Spanish clitic climbing, en Synchronic and diachronic approaches to linguistic variation and change. Ed. T. J. Walsh. Georgetown University Press, Washington, DC, pp. 227-250.

Navarro Correa, M. 1989. "La alternancia -ral-se y -ral-ría en el habla de Valencia (Venezuela)", LEA, 11, 117-123.

— 1992. "Valoración social de algunas frases verbales en el habla de Valencia", $L E A, 14,97-106$.

OCAMPO, F. 1990. "The pragmatics of word order in constructions with a verb and a subject", $H L, 4,87-128$.

Prince, E. F. 1981. "Toward a taxonomy of given-new information", en Radical pragmatics. Ed. P. Cole. Academic Press, New York-LondonToronto-Sydney-San Francisco, pp. 223-255.

Rabanales, A. 1974. "Queísmo y dequeísmo en el español de Chile", en Homenaje a Ángel Rosenblat en sus 70 años: Estudios filológicos y lingüísticos. Inst. Pedagógica, Caracas, pp. 413-444.

Riemsdju, Henk van, y Edwin Williams 1990. Introducción a la teoría gramatical. Trads. L. Guerra Salas y P. Martín Butragueño. Cátedra, Madrid.

Romaine, S. 1981. "On the problem of syntactic variation: A reply to Beatriz Lavandera and William Labov". Working Papers in Sociolinguistics. 
Sociolinguistic Working Paper 82. Southwest Educational Development Laboratory, Austin, TX.

SANKOFF, D. 1988. "Sociolinguistics and syntactic variation", en Linguistics: The Cambridge survey. T. 4: Language: The sociocultural context. Ed. F. J. Newmeyer. Cambridge University Press, Cambridge, pp. 140-161.

Serrano, M. J. 1993. "Aspectos teóricos de la variación sintáctica", comunicación presentada al XI Congreso Internacional de la ALFAL, celebrado del 11 al 16 de abril de 1993 en Veracruz, México.

Silva-Corvalán, Carmen 1989. Sociolingüística. Teoría y análisis. Alhambra, Madrid.

WeINER, E. J., y W. LABOV 1983. "Constraints on the agentless passive", $J L, 19,29-58$. 
